\title{
Numerical Study of Energy Deposition Requirements for Aerodynamic Control of Hypersonic Vehicles
}

\author{
Nicholas J. Bisek* and Iain D. Boyd ${ }^{\dagger}$ \\ Department of Aerospace Engineering, University of Michigan, Ann Arbor, MI, 48109, USA \\ and \\ Jonathan Poggie $\ddagger$ \\ Air Force Research Laboratory, Wright-Patterson AFB, OH, 45433-7512, USA
}

\begin{abstract}
Plasma actuators and various forms of volumetric energy deposition have received a good deal of research attention recently as a means of hypersonic flight control. An open question remains as to whether the required power expenditures for such devices can be achieved for practical systems. To address this issue, a numerical study is carried out for Mach 12 flow over a blunt nose elliptic cone to determine the amount of energy deposition necessary for flight control. Energy deposition is simulated by means of a phenomenological dissipative heating model. Validation studies of the flow simulation code in the absence of energy deposition are presented for a Mach 8 elliptic cone flow and a Mach 14 blunt elliptic cone. A parametric study of the effects of energy deposition is carried out for two blunt-nosed elliptic cone configurations: a $3 \mathrm{~m}$ long cone at Mach 12.6 and $40 \mathrm{~km}$ altitude, and a $0.2 \mathrm{~m}$ long cone at Mach 14.2 and roughly $42 \mathrm{~km}$ effective altitude. Three different volumetric energy deposition patterns are considered: a spherical pattern, a 'pancake' pattern (oblate spheroid), and a 'bean' pattern (prolate spheroid). The effects of energy deposition are seen to be relatively independent of these patterns. For the Mach $\mathbf{1 2 . 6}$ cone case, the pitching moment generated by energy deposition is $10-30 \%$ of that generated by a $0.05 \mathrm{~m}^{2}$ mechanical flap at $2^{\circ}$ deflection. For the Mach 14.2 case, the corresponding figure of merit is on the order of $1000 \%$. The effectiveness of volumetric energy deposition for flight control appears to scale strongly on the nondimensional parameter $Q /\left(\rho_{\infty} u_{\infty}^{3} L^{2}\right)$, and additional computations are warranted to explore this effect.
\end{abstract}

\section{Nomenclature}

$\rho \quad=$ mass density

$\mu \quad=$ coefficient of viscosity

$\mathbf{u} \quad=$ velocity vector $(u, v, w)$

$x, y, z=$ streamwise, spanwise, and transverse coordinates

$i, j, k=$ computational grid indices along the axial, radial, and circumferential directions

$a, b, c=$ the equatorial radii and the polar radius of an ellipsoid

$\phi \quad=$ angle between the $\mathrm{x}$-axis of the ellipsoid and the $\mathrm{x}$-axis of the flow

$\theta \quad=$ ramp angle

$A \quad=$ surface area of grid cell

$\mathbf{n} \quad=$ normal vector

$p \quad=$ pressure

$\tau \quad=$ viscous stress

$E \quad=$ total energy per volume

$\mathbf{q}=$ heat flux (translational, rotational, and vibrational)

${ }^{*}$ Graduate Student, Student Member AIAA

${ }^{\dagger}$ Professor, Associate Fellow AIAA

${ }^{\ddagger}$ Senior Aerospace Engineer, AFRL/VAAC. Bldg. 146 Rm. 225, 2210 Eighth St. Associate Fellow AIAA

This material is declared a work of the U.S. Government and is not subject to copyright protection in the United States. 


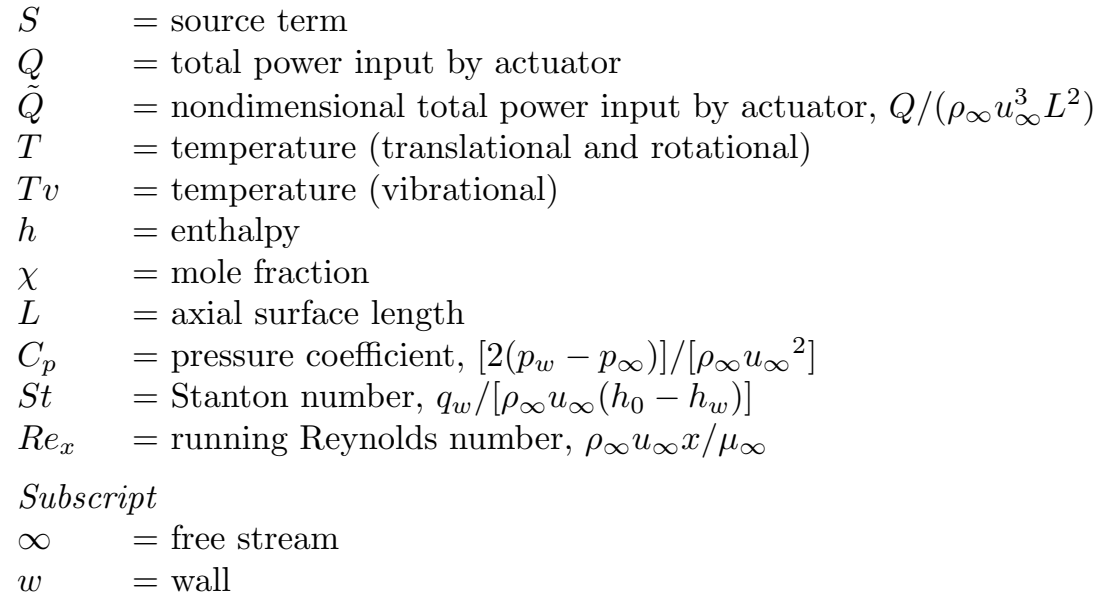

\section{Introduction}

Aerodynamic control and drag reduction are major challenges for hypersonic vehicle designers. A good deal of research attention has recently focused on hypersonic plasma interactions and plasma flow control to explore ways of confronting these challenges. ${ }^{3,4}$

Minimizing drag in vehicle design leads to long thin bodies with sharp leading edges. This constrains the materials available for the vehicle's Thermal Protection System (TPS) because there is a required minimum thickness which may not be achieved for a given vehicle configuration. In addition, small defects in the production of the sharp edges can result in serious or even catastrophic problems for the TPS. ${ }^{5}$ Blunting the leading edge reduces these drawbacks but results in a much larger wave drag. ${ }^{6}$ Recent experimental and computational research by Shang et al. ${ }^{7}$ has investigated ways of reducing drag on blunt nose bodies by means of plasma injection, while research by Kremeyer et al. ${ }^{8}$ and $\mathrm{Yan}^{9}$ focused on drag reduction and flow control using laser deposition (filamentation) ahead of conic and spherical geometries.

In addition to the design constraints, extreme heat, and pressure, traditional control surfaces (flaps) need to be positioned away from the center of gravity to extend the maneuverability of the vehicle. The location of such flaps is limited because the bow shock surrounding the vehicle will impinge on surfaces that extend beyond the shock envelope. This results in extreme pressure and heat transfer rates at the impingement point. As such, vehicle configurations tend to be streamlined with minimal protrusions from the fuselage. Mechanically driven flaps require clearance below the surface of the flap to provide space for the flap control arm and a strong attachment point to push from. In addition, there is a small gap in the TPS as the flap extends out to deflect the flow. This gap is difficult to protect and can cause heat related damage to the vehicle.

Plasma actuators are advantageous over mechanical controllers because they do not have moving parts, can be located either in or beneath the TPS, and use an electric power source. This extends the range of possible locations for the actuator and allows for multiple actuators to be powered by a central energy source. They can potentially be turned on and off very rapidly, and should have a minimal aerothermal penalty when turned off. Plasma actuators can serve multiple roles. They can be used to provide steering moments, ${ }^{10}$ changes in vehicle lift, ${ }^{11}$ control of flow separation, ${ }^{12,13}$ and local heat load mitigation. ${ }^{14}$

The primary objective of this research effort is to determine whether a useful degree of flight control can be achieved with practical levels of energy deposition by investigating the effects of energy deposition on a realistic hypersonic vehicle and its surrounding flow-field. In the following, we first present code validation studies using experimental data in the literature. Having successfully validated LeMANS, the code is applied to investigate plasma-based aerodynamic control.

\section{Method}

Flow-field results are obtained using Computational Fluid Dynamics (CFD) to solve the Navier-Stokes equations. The CFD computations are executed using the Michigan Aerothermodynamic Navier-Stokes 
(LeMANS) code which was developed at the University of Michigan. ${ }^{15,16}$

LeMANS is a general 2D/axisymmetric/3D, parallel, unstructured finite-volume CFD code. The numerical fluxes between cells are discretized using a modified Steger-Warming Flux Vector Splitting (FVS) scheme, except near shock waves. In these regions the original Steger-Warming FVS scheme is used. LeMANS employs a two-temperature model to account for thermal-nonequilibrium and a standard finite rate chemistry model for non-equilibrium chemistry. The two temperature model assumes that a single temperature $T$ accounts for the translational and rotational energy modes of all species while the vibrational energy mode is accounted for by a separate temperature $T v$.

The usual conservation equations are solved:

$$
\begin{gathered}
\frac{\partial \rho}{\partial t}+\nabla \cdot(\rho \mathbf{u})=0 \\
\frac{\partial \rho \mathbf{u}}{\partial t}+\nabla \cdot\left(\rho \mathbf{u}^{2}+p \delta_{i j}-\tau\right)=0 \\
\frac{\partial E}{\partial t}+\nabla \cdot((E+p) \mathbf{u}-\tau \cdot \mathbf{u}-\mathbf{q})=S
\end{gathered}
$$

LeMANS assumes the fluid is continuous and Newtonian. It also assumes Stokes' hypothesis when determining the viscous stresses.

$$
\tau_{i j}=\mu\left(\frac{\partial u_{j}}{\partial x_{i}}+\frac{\partial u_{i}}{\partial x_{j}}\right)-\frac{2}{3} \mu \nabla \cdot \mathbf{u} \delta_{i j}
$$

A thermal actuator is considered as the plasma control device in this study. It is represented by a phenomenological model of dissipative heating. This model is accounted for in the Navier-Stokes equations by the addition of a source term $S$ to the right side of energy equation (3). The shape, strength, and location of the actuator are modeled using exponential decay of an ellipsoidal region. ${ }^{17}$

$$
\begin{aligned}
S & =\frac{Q}{\pi^{3 / 2} a \cdot b \cdot c} e^{\left(-\left(\frac{\hat{x}}{a}\right)^{2}-\left(\frac{\hat{y}}{b}\right)^{2}-\left(\frac{\hat{z}}{c}\right)^{2}\right)} \\
\hat{x} & =\left(x-x_{0}\right) \cos \phi-\left(z-z_{0}\right) \sin \phi \\
\hat{y} & =\left(y-y_{0}\right) \\
\hat{z} & =\left(x-x_{0}\right) \sin \phi+\left(z-z_{0}\right) \cos \phi
\end{aligned}
$$

Variables $a$ and $b$ are the equatorial radii (along the $x$ and $y$ axes) and $c$ is the polar radius (along the $z$-axis). The angle $\phi$ is the angle between the $\mathrm{x}$-axis of the ellipsoid and the x-axis of the flow. Coordinates $\left(x_{0}, y_{0}, z_{0}\right)$ represent the centroid of the ellipsoid. Note that $Q$ represents the total power deposited in the flow and $\iiint_{-\infty}^{\infty} S d x d y d z=Q$

The simulations are performed using second-order accurate discretization and carry double precision arithmetic throughout. Thermal equilibrium and a five species air chemistry model $\left(\mathrm{N}_{2}, \mathrm{O}_{2}, \mathrm{NO}, \mathrm{N}\right.$, and $O)$ are used in the simulations presented.

\section{Results}

\section{III.A. 3D Sharp Elliptic Cone}

Three dimensional calculations are carried out for a Mach 8 sharp elliptic cone originally studied experimentally by Kimmel et al.. ${ }^{1,18}$ The cone was mounted parallel to the freestream and consisted of a 2:1 aspect ratio, a half angle along the major axis of $14^{\circ}$, and a length $L=1.016 \mathrm{~m}$. It was machined from stainless steel with a $40 \mu \mathrm{m}$ nose radius and surface roughness less than $0.81 \mu \mathrm{m}$. The flow conditions are listed in Table 1.

A structured grid is generated because it is known to produce better results in regions near the surface of the body and through a shock. ${ }^{19}$ One quarter of the geometry is used in the simulation because planes of symmetry exist along the major and minor axes. The $40 \mu \mathrm{m}$ nose radius is accounted for along the tip's minor axis, resulting in a $80 \mu \mathrm{m}$ radius along the major axis because of the elliptic geometry. 


\begin{tabular}{|c|rl|}
\hline Parameter & Value \\
\hline \hline $\mathrm{M}$ & 7.93 & \\
$u_{\infty}$ & 1175.0 & $\mathrm{~m} / \mathrm{s}$ \\
$\mathrm{T}_{\infty}$ & 54.6 & $\mathrm{~K}$ \\
$\mathrm{~T}_{w}$ & 303.0 & $\mathrm{~K}$ \\
$\mathrm{~T}_{0}$ & 728.0 & $\mathrm{~K}$ \\
$\mathrm{p}_{\infty}$ & 165.0 & $\mathrm{~Pa}$ \\
$\rho_{\infty}$ & 0.010533 & $\mathrm{~kg} / \mathrm{m}^{3}$ \\
$\mu_{\infty}$ & $3.77 \times 10^{-6}$ & $\mathrm{~kg} / \mathrm{m} \cdot \mathrm{s}$ \\
$\operatorname{Re}_{L}$ & $3.33 \times 10^{6}$ & \\
\hline
\end{tabular}

Table 1. Flow conditions for the experiment of Kimmel et al. ${ }^{1,18}$

The model is aligned with the $\mathrm{x}$-axis in the axial direction, the $\mathrm{y}$-axis in the horizontal direction, and the $\mathrm{z}$-axis in the vertical direction. A cylindrical coordinate system is also employed with $\theta=0^{\circ}$ at the top centerline of the model (z-axis) and $\theta=90^{\circ}$ at the leading edge (y-axis) as seen in Figure 1.

A gradual increase in grid spacing is used along the conic body with the smallest spacing near the tip. Radial points are algebraically spaced to increase the number of points close to the body. Grid points are equally spaced along the circumference. As a result, cell clustering occurs near the surface and the tip of the body. A grid independence study is conducted with $i \times j \times k$ grid dimensions changing from $330 \times 40 \times 30$ (coarse), to $440 \times 50 \times 40$ (medium), to $550 \times 60 \times 50$ (fine). Figure 2 shows very little change in $C p$ or $S t$ between the medium and fine grids, therefore the medium grid $(440 \times 50 \times 40)$ is considered grid independent and used in the rest of the analysis.

Cross-sectional slices of the computed surface conditions are extracted to match the locations of the experimental measurements. Figure 3(a) shows the non-dimensional pressure along the circumference of the body at $\mathrm{x} / \mathrm{L}=0.625$. The pressure is relatively constant from the top centerline $\left(\theta=0^{\circ}\right)$ to the shoulder $\left(\theta=45^{\circ}\right)$, followed by a noticeable rise between the shoulder and the leading edge $\left(\theta=90^{\circ}\right)$. This behavior was also observed by Burke for a Mach 10 flow. ${ }^{20}$ Kimmel et al. also provided computational results from a parabolized Navier-Stokes (PNS) solver ${ }^{1,18}$ which are included in the figures as an additional reference.

Although the cone is sharp, the formation of the boundary layer at its tip results in a sharp rise in pressure and temperature near the stagnation point. This rise in pressure can be seen in Figure 3(b) for two different rays. The pressure quickly relaxes as the flow proceeds along the rest of the cone due to the viscous interaction.

The high length Reynolds number $\left(R e_{L}\right)$ and overall length of the model cause the flow to transition to turbulence as it proceeds along the body. LeMANS does not currently have a turbulence model implemented, so numerical results in the transition and turbulent regions should be disregarded. Plots of the Stanton number as a function of Reynolds number are presented in Figures 4(a), 4(b) and 4(c) for $\theta=0^{\circ}, 45^{\circ}$, $88^{\circ}$. In all three plots, the flow starts laminar and then transitions to turbulent as it proceeds along the body. The measured data were for $R e_{L}=1.7 \times 10^{6}$ and $6.6 \times 10^{6}$, whereas the case run by LeMANS has $R e_{L}=3.3 \times 10^{6}$. As observed in the figures, the length Reynolds number does not affect the Stanton number in the laminar region for these cases.

\section{III.B. 3D Blunt Elliptic Cone}

A second three dimensional validation study is performed on a Mach 14 blunt elliptic cone originally studied experimentally by Nowlan et al.. ${ }^{2}$ The model was mounted parallel to the freestream and had a 2:1 aspect ratio, a half angle along the major axis of $10^{\circ}$, and a length $L=0.21 \mathrm{~m}$. Details of cone geometry are provided in Fig. 5. The flow conditions are listed in Table 2.

A structured grid is generated following the same procedures and coordinate system as the sharp elliptic cone. A grid independence study is conducted with $i \times j \times k$ grid dimensions changing from $150 \times 30 \times 30$ (coarse), to $300 \times 60 \times 60$ (medium), to $380 \times 80 \times 80$ (fine). Figure 6 shows very little change in $C p$ or $S t$ between the medium and fine grids, therefore the medium grid $(300 \times 60 \times 60)$ is considered grid independent and used in the rest of the analysis. 


\begin{tabular}{|c|c|c|}
\hline Parameter & \multicolumn{2}{|c|}{ Value } \\
\hline Mach & 14.15 & \\
\hline$u_{\infty}$ & 2184.8 & $\mathrm{~m} / \mathrm{s}$ \\
\hline $\mathrm{T}_{\infty}$ & 59.3 & $\mathrm{~K}$ \\
\hline $\mathrm{T}_{w}$ & 294.4 & K \\
\hline $\mathrm{T}_{0}$ & 2105.0 & K \\
\hline $\mathrm{p}_{\infty}$ & 51.0 & $\mathrm{~Pa}$ \\
\hline$\rho_{\infty}$ & 0.002995 & $\mathrm{~kg} / \mathrm{m}^{3}$ \\
\hline$\mu_{\infty}$ & $4.3 \times 10^{-6}$ & $\mathrm{~kg} / \mathrm{m} \cdot \mathrm{s}$ \\
\hline $\operatorname{Re}_{L}$ & $3.17 \times 10^{5}$ & \\
\hline
\end{tabular}

Table 2. Flow conditions for Run 15 of the Nowlan et al. experiment. ${ }^{2}$

Figure 7 shows the non-dimensional pressure along the circumference of the body at two axial locations and along two rays. Following a similar trend as the sharp cone observations, the pressure is relatively constant from the top centerline to the shoulder, followed by a gradual rise between the shoulder and the leading edge. The variation in pressure distribution along the rays is much more dramatic compared to the sharp cone because the blunt tip results in a strong detached bow shock and, consequently, a large stagnation region.

Stanton number distributions in Fig. 8 show that LeMANS follows the same trends as those observed in the measurements. Overall, LeMANS effectively demonstrates its capability of accurately computing three dimensional hypersonic flows.

\section{III.C. Realistic Hypersonic Geometry}

The blunt elliptic cone geometry is selected to represent a realistic hypersonic vehicle, with $L=3 \mathrm{~m}$ set as the representative vehicle length. Assuming the vehicle has constant material density, its center of gravity $(\mathrm{CG})$ is located $1.95 \mathrm{~m}$ from the tip along the $\mathrm{x}$-axis $(\mathrm{x} / \mathrm{L}=0.65)$. The model is simulated in air at $40 \mathrm{~km}$ altitude, with a freestream velocity of $4000 \mathrm{~m} / \mathrm{s}$. The complete flow conditions are provided in Table 3 .

\begin{tabular}{|c|c|c|}
\hline Parameter & \multicolumn{2}{|c|}{ Value } \\
\hline Mach & 12.6 & \\
\hline$u_{\infty}$ & 4000.0 & $\mathrm{~m} / \mathrm{s}$ \\
\hline $\mathrm{T}_{\infty}$ & 250.4 & $\mathrm{~K}$ \\
\hline $\mathrm{T}_{w}$ & 300.0 & K \\
\hline $\mathrm{T}_{0}$ & 8300.0 & K \\
\hline $\mathrm{p}_{\infty}$ & 289.2 & $\mathrm{~Pa}$ \\
\hline$\rho_{\infty}$ & 0.003995 & $\mathrm{~kg} / \mathrm{m}^{3}$ \\
\hline$\mu_{\infty}$ & $1.6 \times 10^{-5}$ & $\mathrm{~kg} / \mathrm{m} \cdot \mathrm{s}$ \\
\hline $\operatorname{Re}_{L}$ & $3.0 \times 10^{6}$ & \\
\hline
\end{tabular}

Table 3. Flow conditions for Mach 12.6 air flow at an altitude of $40 \mathrm{~km}$.

A grid independence study is conducted with $i \times j \times k$ grid dimensions changing from $150 \times 30 \times 30$ (coarse), to $300 \times 60 \times 60$ (medium), to $380 \times 80 \times 80$ (fine), to $400 \times 80 \times 120$ (very fine). Figure 9 shows very little change in $C p$ or $S t$ between the fine and very fine grids. Grid independence is achieved with the $380 \times 80 \times 80$ (fine) grid and is used in the following simulations that include energy deposition.

\section{III.C.1. Nominal Force Requirement}

An estimate of the pitching moment produced by a mechanical flap attached to this nominal vehicle is found by assuming a $2^{\circ}$ flap with a cross-sectional area of $0.05 \mathrm{~m}^{2}(0.5 \mathrm{~m} \times 0.1 \mathrm{~m})$ is attached along the vehicle's 
top centerline as illustrated in Fig. 10. Figure 11 plots a cross-sectional slice of Mach number contours where the beginning of the flap protrudes from the body. Setting $\mathrm{M}_{\infty}=1.5$ and $\theta=2^{\circ}$, oblique shock theory is used to find $p_{\text {flap }} \approx 1000 \mathrm{~Pa}$. This results in a pitching moment of about $50 \mathrm{~N}$-m.

\section{III.C.2. Parametric Study Conditions}

To limit the scope of the problem, three volumetric deposition shapes are selected. Namely a sphere, pancake (oblate spheroid), and bean (prolate spheroid) are employed such that the volume of the ellipsoid $(V=4 / 3 \pi a b c)$ remains constant. The values used are listed in Table 4.

\begin{tabular}{|c|c|c|c|}
\hline & $a$ & $b$ & $c$ \\
\hline \hline Sphere & $0.007 \mathrm{~m}$ & $0.007 \mathrm{~m}$ & $0.007 \mathrm{~m}$ \\
Pancake & $0.01852 \mathrm{~m}$ & $0.01852 \mathrm{~m}$ & $0.001 \mathrm{~m}$ \\
Bean & $0.001852 \mathrm{~m}$ & $0.1 \mathrm{~m}$ & $0.001852 \mathrm{~m}$ \\
\hline
\end{tabular}

Table 4. Deposition geometry parameters.

The centroid of the deposition is positioned along the top centerline $\left(y_{0}=0\right)$ and must be at least three characteristic length scales $(\sigma)$ away from the surface of the body to ensure the entire deposition is deposited into the flow-field $\left(\iiint_{-3 \sigma}^{3 \sigma} S d x d y d z=0.9999 * Q\right)$. This distance is the minimal length from the centroid of a spherical energy deposition to the surface of the body and is denoted, $\mathrm{f}$, in Figure 12. With $\mathrm{f}=3 \sigma=3 \mathrm{a}=$ $0.021 \mathrm{~m}$, the values of $z_{0}$ and $\phi$ can be determined for a given $x_{0}$ by enforcing equation (7). This determines the location of $\left[x_{1}, z_{1}\right]$ and its outward normal unit vector $\vec{n}$. Equation (9) is used to determine $\phi$ so the cross-section of the deposition is parallel to the body as illustrated in Figure 13 for a pancake deposition.

$$
\begin{gathered}
x_{0}=x_{1}+\mathrm{f} \cdot\left\|n_{x_{1}}\right\| \\
z_{0}=z_{1}+\mathrm{f} \cdot\left\|n_{z_{1}}\right\| \\
\phi=\tan ^{-1}\left(\left\|\frac{n_{x_{1}}}{n_{z_{1}}}\right\|\right)
\end{gathered}
$$

Using the Mach 5 flat plate experiment originally studied by Kimmel et al. ${ }^{4,11,13,21-26}$ and recent power generation experiments, ${ }^{27}$ realistic power input is assumed to lie in the range of $1000 \mathrm{~W}$ to $15,000 \mathrm{~W}$. The deposition is positioned near the vehicle tip to maximize the distance from the center of gravity (CG). Variation in the input power, $Q$, and the distance along the body, $\mathrm{x} / \mathrm{L}$, leads to the test matrix shown in Table 5 .

\begin{tabular}{|l|c|c||c|c|c||c|c|c|}
\hline & $\mathrm{Q}[\mathrm{W}]$ & $\mathrm{x} / \mathrm{L}$ & & $\mathrm{Q}[\mathrm{W}]$ & $\mathrm{x} / \mathrm{L}$ & & $\mathrm{Q}[\mathrm{W}]$ & $\mathrm{x} / \mathrm{L}$ \\
\hline \hline Sphere & 1000 & $5 \%$ & Pancake & 1000 & $5 \%$ & Bean & 1000 & $5 \%$ \\
Sphere & 4000 & $5 \%$ & Pancake & 4000 & $5 \%$ & Bean & 4000 & $5 \%$ \\
Sphere & 15000 & $5 \%$ & Pancake & 15000 & $5 \%$ & Bean & 15000 & $5 \%$ \\
Sphere & 1000 & $10 \%$ & Pancake & 1000 & $10 \%$ & Bean & 1000 & $10 \%$ \\
Sphere & 4000 & $10 \%$ & Pancake & 4000 & $10 \%$ & Bean & 4000 & $10 \%$ \\
Sphere & 15000 & $10 \%$ & Pancake & 15000 & $10 \%$ & Bean & 15000 & $10 \%$ \\
\hline
\end{tabular}

Table 5. Parametric study for three different deposition shapes.

\section{III.C.3. Parametric Study Results}

The total amount of energy deposited into the flow is characterized by the nondimensional total energy deposition value $\tilde{Q}$. For the cases in the study $\tilde{Q}=4.3 \times 10^{-7}, 1.7 \times 10^{-6}$, and $6.5 \times 10^{-6}$ for $\mathrm{Q}=1000$ $\mathrm{W}, 4000 \mathrm{~W}, 15000 \mathrm{~W}$ respectively. This parameter provides some information on vehicle and application scaling. 


$$
\tilde{Q}=\frac{Q}{\rho_{\infty} u_{\infty}^{3} L^{2}}
$$

The axial location of the energy deposition is apparent after investigating the pressure coefficient and Stanton number along the top centerline $\left(\theta=0^{\circ}\right)$ for the three shapes in Figure 14. Although there is a slight increase in the Stanton number, it is accompanied by a noticeable rise in the pressure coefficient, particularly in the sphere and pancake depositions. The total forces acting on the body in the Cartesian coordinate system are found by multiplying the combined stress tensor and pressure matrices by the corresponding area vector.

$$
\left\{\begin{array}{l}
F_{x} \\
F_{y} \\
F_{z}
\end{array}\right\}_{i j}=\left\{\left[\begin{array}{lll}
\tau_{x x} & \tau_{x y} & \tau_{x z} \\
\tau_{y x} & \tau_{y y} & \tau_{y z} \\
\tau_{z x} & \tau_{z y} & \tau_{z z}
\end{array}\right]-\left[\begin{array}{ccc}
p & 0 & 0 \\
0 & p & 0 \\
0 & 0 & p
\end{array}\right]\right\}\left[\begin{array}{l}
n_{x} \\
n_{y} \\
n_{z}
\end{array}\right] A
$$

With the local force known, the pitching moment is determined by multiplying the downward force $\left(F_{z_{i j}}\right)$ by the moment arm $\left(\Delta x=x_{i j}-x_{C G}\right)$. For each scenario, the pitching moment is compared to the baseline and then normalized by the effective pitching moment due to the mechanical flap found previously $\left(M_{p_{\text {flap }}}=50 \mathrm{Nm}\right)$. Figure 15 plots the normalized change in total pitching moment for each of the shapes. The points are fitted with a parametric spline because of their assumed non-linearity. The plots show the energy deposition is unable to provide the same level of control authority as the mechanical flap. In addition, while the shape of the deposition appears to have noticeable effects on the local pressure coefficient and Stanton number, it does not appear to have a large impact on the overall change in the pitching moment.

\section{III.D. Small Blunt Elliptic Cone With Energy Deposition}

The effects of energy deposition are also simulated for a few cases using the $L=0.2 \mathrm{~m}$ blunt elliptic cone with freestream conditions found in Table 2. Using the smaller geometry and the higher altitude freestream conditions increases the non-dimensional total energy deposition value $\tilde{Q}$ by several orders of magnitude. For the cases run, $\tilde{Q}=4.0 \times 10^{-4}, 8.0 \times 10^{-4}$, and $1.6 \times 10^{-3}$ for $\mathrm{Q}=500 \mathrm{~W}, 1000 \mathrm{~W}$, and $2000 \mathrm{~W}$ respectively.

The deposition is modeled as an oblate spheroid (Pancake), though its geometry is not identical to the one run in the $L=3 \mathrm{~m}$ parametric study. Table 6 lists the values used to represent the energy deposition volume and location. The energy deposition is positioned near the bow shock $(\mathrm{x} / \mathrm{L}=0.15 \mathrm{~m})$.

\begin{tabular}{|c|c|c|c|c|c|c|}
\hline$x_{0}$ & $y_{0}$ & $z_{0}$ & $a$ & $b$ & $c$ & $\theta$ \\
\hline \hline $3 \mathrm{~cm}$ & $0.0 \mathrm{~cm}$ & $1.7 \mathrm{~cm}$ & $0.3 \mathrm{~cm}$ & $0.4 \mathrm{~cm}$ & $0.1 \mathrm{~cm}$ & $0^{\circ}$ \\
\hline
\end{tabular}

Table 6. The location of energy deposition for Mach 14 blunt elliptic cone.

Unlike the larger geometry cases, the energy deposition on the smaller blunt cone results in a large temperature rise at and downstream of the deposition as illustrated in Figure 16 for $Q=1000 \mathrm{~W}$ deposition. This results in a strong heat transfer penalty, but gives rise to a dramatic increase in the local peak pressure coefficient distribution as seen in Figure 17. The pitching moment due to the flap is found following the layout covered in subsection III.C.1 with the flap geometry scaled to match the previous flap dimensions $\left(M_{p_{\text {flap }}}=4 \times 10^{-3} \mathrm{Nm}\right)$. The effective pitching moment, shown in Figure 18, indicates that energy deposition is an viable replacement for the mechanical flap under these conditions. These results are combined with those obtained for the large blunt elliptic cone using the using the non-dimensional total energy deposition parameter $\tilde{Q}$. Figure 19 shows a strong correlation between $\tilde{Q}$ and the effective pitching moment and suggests that energy deposition is a viable replacement for a mechanical flap when $\tilde{Q}>1 \times 10^{-4}$.

\section{Conclusions}

The Michigan Aerothermodynamic Navier-Stokes (LeMANS) code was successfully validated for threedimensional blunt and sharp elliptic cones. In addition, a phenomenological heating model was implemented to investigate whether a practical level of control could be achieved for a realistic hypersonic vehicle. A parametric study was completed investigating the shape, location, and total amount of energy volumetrically deposited into the flow-field for two blunt-nosed elliptic cone configurations. The effectiveness of 
volumetric energy deposition for flight control appeared to scale strongly on the nondimensional parameter $Q /\left(\rho_{\infty} u_{\infty}^{3} L^{2}\right)$, warranting additional computations to explore this effect.

\section{Future Work}

In future studies, we will consider the potential additional benefits of electromagnetic fields. The addition of the magnetohydrodynamics (MHD) equations to LeMANS by means of a current continuity equation module will provide additional design tools to model design configurations that incorporate the use of a magnetic field to help with flow control. The Mach 5 flat plate experiment mentioned previously had some experiments measuring the effects of a magnetic field on the flow, with and without energy deposition from the plasma actuator. ${ }^{13,22}$ Their work showed an additional increase in resulting forces, when the plasma actuator was on. This experiment will be used to verify the addition of the MHD module.

\section{Acknowledgments}

The authors are indebted to the Michigan/AFRL/Boeing Collaborative Center in Aeronautical Sciences which provides funding to the first author. The first author would like to thank Leonardo Scalabrin for numerous discussions about LeMANS and Dr. Roger Kimmel for his valuable discussions on the subject. The generous use of the University of Michigan's Center for Advanced Computing and the Aeronautical Systems Center Major Shared Resource Center (ASC MSRC) were indispensable to this investigation and are greatly appreciated.

\section{References}

${ }^{1}$ Kimmel, R. L., Poggie J., and Schwoerke S. N., "Laminar-Turbulent Transition in a Mach 8 Elliptic Cone Flow," AIAA Journal, Vol. 37, No. 9, September 1999, pp. 1080-1087.

${ }^{2}$ Nowlan, D., Burke, A., and Bird, K., "Pressure and Heat Transfer Distribution on ASD Elliptic Cone (W3) and ASD Sortie (W4) in the CAL 48-Inch Hypersonic Shock Tunnel," CAL Report AM-1800-Y-2, December 1963. ASC 942649

${ }^{3}$ Fomin, V. M., Tretyakov, P. K., and Taran J.-P., "Flow Control using Various Plasma and Aerodynamic Approaches," Aerospace Science and Technology, Vol. 8, No. 5, July 2004, pp. 411-421.

${ }^{4}$ Shang, J. S., Surzhikov, S. T., Kimmel, R., Gaitonde, D., Menart, J., and Hayes, J., "Mechanisms of Plasma Actuators for Hypersonic Flow Control," Progress in Aerospace Sciences, Vol. 41, No. 8, November 2005, pp. 642-668.

${ }^{5}$ Mason, W. H., and Lee, J., "Aerodynamically Blunt and Sharp Bodies," AIAA paper 1992-2727, American Institute of Aeronautics and Astronautics, Palo Alto CA, June 1992.

${ }^{6}$ Santos, W. F. N., and Lewis, M. J., "Aerothermodynamic Performance Analysis of Hypersonic Flow on Power Law Leading Edges," Journal of Spacecraft and Rockets, Vol. 42, No. 4, July-August 2005, pp. 588-597.

${ }^{7}$ Shang, J. S., Hayes, J., and Menart, J., "Hypersonic Flow over a Blunt Body with Plasma Injection," Journal of Spacecraft and Rockets, Vol. 39, No. 3, May-June 2002, pp. 367-375.

${ }^{8}$ Kremeyer, K., Sebastian, K., and Shu, C.-W., "Computational Study of Shock Mitigation and Drag Reduction by Pulsed Energy Lines," AIAA Journal, Vol. 44, No. 8, August 2006.

${ }^{9}$ Yan, H., and Gaitonde, D., "Control of Edney IV Interaction by Energy Pulse," AIAA Paper 2006-562, American Institute of Aeronautics and Astronautics, Reno NV, January 2006.

${ }^{10}$ Girgis, I. G., Shneider, M. N., Macheret, S. O., Brown, G. L., and Miles, R. B., "Creation of Steering Moments in Supersonic Flow by Off-Axis Plasma Heat Addition," AIAA paper 2002-0129, American Institute of Aeronautics and Astronautics, Reno NV, January 2002.

${ }^{11}$ Menart, J., Stanfield, S., Shang, J., Kimmel, R., and Hayes, J., "Study of Plasma Electrode Arrangements for Optimum Lift in a Mach 5 Flow," AIAA paper 2006-1172, American Institute of Aeronautics and Astronautics, Reno NV, January 2006.

${ }^{12}$ Updike, G. A., Shang, J. S., and Gaitonde, D. V., "Hypersonic Separated Flow Control Using Magneto-Aerodynamic Interaction," AIAA paper 2005-164, American Institute of Aeronautics and Astronautics, Reno NV, January 2005.

${ }^{13}$ Kimmel, R. L., Hayes, J. R., Crafton., J. W., Fonov, S. D., Menart, J., and Shang, J., "Surface Discharge for High-Speed Boundary Layer Control," AIAA paper 2006-710, American Institute of Aeronautics and Astronautics, Reno NV, January 2006.

${ }^{14}$ Miles, R. B., Macheret, S. O., Shneider, M. N., Steeves, C., Murray, R. C., Smith, T., and Zaidi, S. H., "Plasma-Enhanced Hypersonic Performance Enabled by MHD Power Extraction," AIAA paper 2005-561, American Institute of Aeronautics and Astronautics, Reno NV, January 2005.

${ }^{15}$ Scalabrin, L. C., and Boyd, I. D., "Development of an Unstructured Navier-Stokes Solver For Hypersonic Nonequilibrium Aerothermodynamics," AIAA Paper 2005-5203, American Institute of Aeronautics and Astronautics, Toronto Canada, June 2005.

${ }^{16}$ Scalabrin, L. C., and Boyd, I. D., "Numerical Simulation of Weakly Ionized Hypersonic Flow for Reentry Configurations," AIAA Paper 2006-3773, American Institute of Aeronautics and Astronautics, San Francisco CA, June 2006.

${ }^{17}$ Poggie J., "Plasma-Based Hypersonic Flow Control," AIAA Paper 2006-3567, American Institute of Aeronautics and Astronautics, San Francisco CA, June 2006. 
${ }^{18}$ Kimmel, R., Klein, M., Schwoerke, S., "Three-Dimensional Hypersonic Laminar Boundary-Layer Computations for Transition Experiment Design," AIAA Journal, Vol. 34 No. 4, July-August 1997, pp. 409-415.

${ }^{19}$ Candler, G., "Unstructured Grid Approaches for Accurate Aeroheating Simulations," AIAA Paper 2007-3959, American Institute of Aeronautics and Astronautics, Miami FL, June 2007.

${ }^{20}$ Burke, G., "Heat Transfer and Pressure Distributions about Sharp and Blunt Elliptic Cones at Angles of Attack and high Mach Numbers," U.S. Air Force Flight Dynamics Lab., TR AFFDL-TR-64-172, Wright-Patterson AFB, OH, May 1965.

${ }^{21}$ Shang, J., Kimmel, R., Hayes, J., and Tyler, C., "Performance of a Low-Density Hypersonic Magneto-Aerodynamic Facility," AIAA Paper 2003-329, American Institute of Aeronautics and Astronautics, Reno NV, January 2003.

${ }^{22}$ Menart, J., Shang, J., Kimmel, R., and Hayes, J., "Effects of Magnetic Fields on Plasmas Generated in a Mach 5 Wind Tunnel," AIAA Paper 2003-4165, American Institute of Aeronautics and Astronautics, Reno NV, January 2003.

${ }^{23}$ Kimmel, R. L., Hayes, J. R., Menart, J. A., and Shang, J., "Effect of Surface Plasma Discharges on Boundary Layers at Mach 5," AIAA Paper 2004-509, American Institute of Aeronautics and Astronautics, Reno NV, January 2004.

${ }^{24}$ Kimmel, R., Hayes, J., Menart, J., and Shang, J., "Effects on Magnetic Fields on Surface Plasma Discharges at Mach 5," AIAA Paper 2004-2661, American Institute of Aeronautics and Astronautics, Reno NV, January 2004.

${ }^{25}$ Menart, J., Henderson, S., Shang, J., Kimmel, R., Hayes, J., "DC Plasma Discharge Effects on a Mach 5 Flow Between Small Plate Electrodes," AIAA Paper 2004-2264, American Institute of Aeronautics and Astronautics, Reno NV, January 2004.

${ }^{26}$ Kimmel, R., Hayes, J., Menart, J., and Shang, J, "Supersonic Plasma Flow Control Experiments," U.S. Air Force Research Lab., TR ARFL-VA-WP-TR-2006-3006, Wright-Patterson Air Force Base, OH, December 2005.

${ }^{27}$ Velocci, A. L. Jr., "A General Atomics-led Team," Aviation Week \& Space Technology, March 19/26, 2007 , pp. 36. 


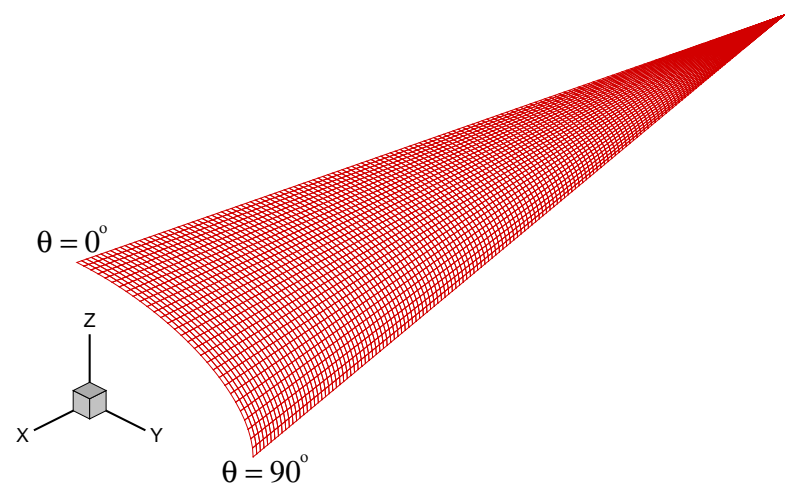

Figure 1. Surface of the sharp elliptic cone grid with both Cartesian and cylindrical coordinate systems.

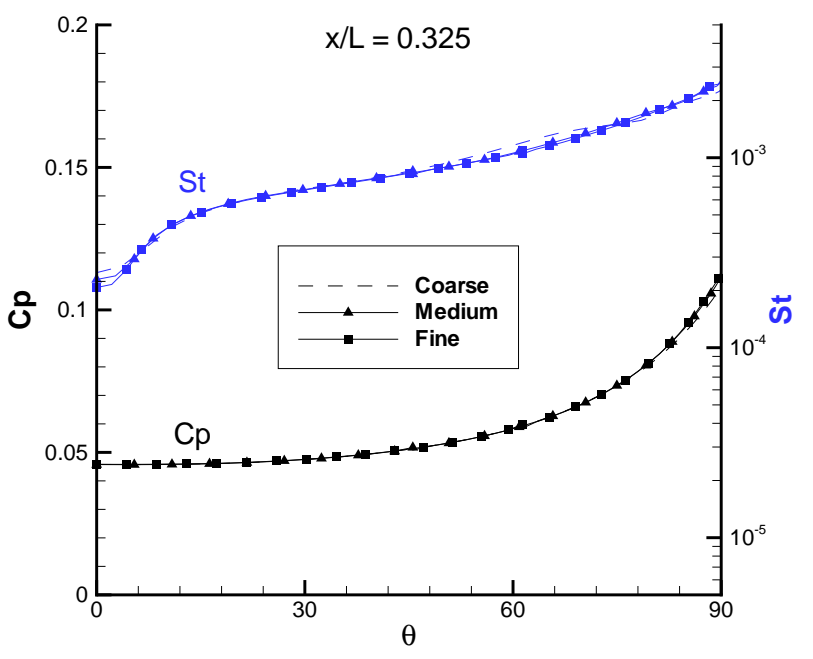

(a) Around the circumference

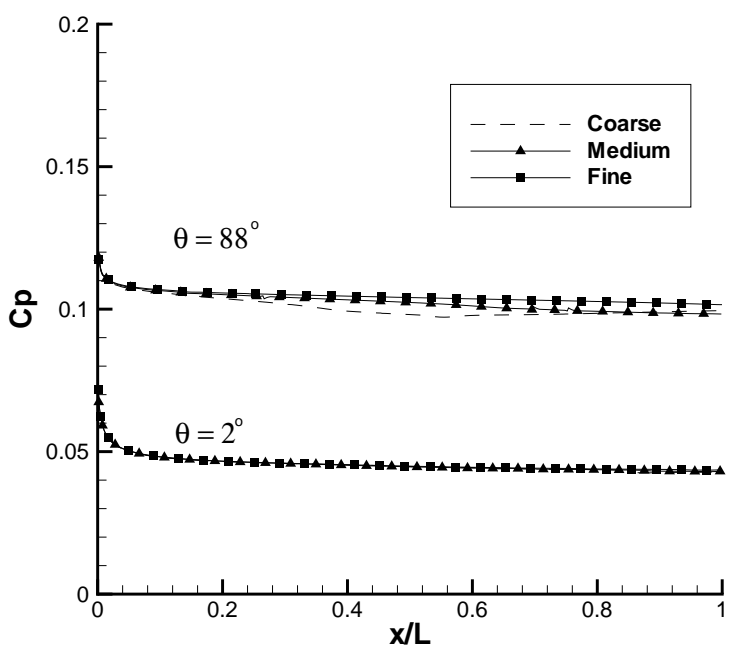

(b) Along rays

Figure 2. Grid independence study for Mach 8 sharp elliptic cone.

10 of 23 


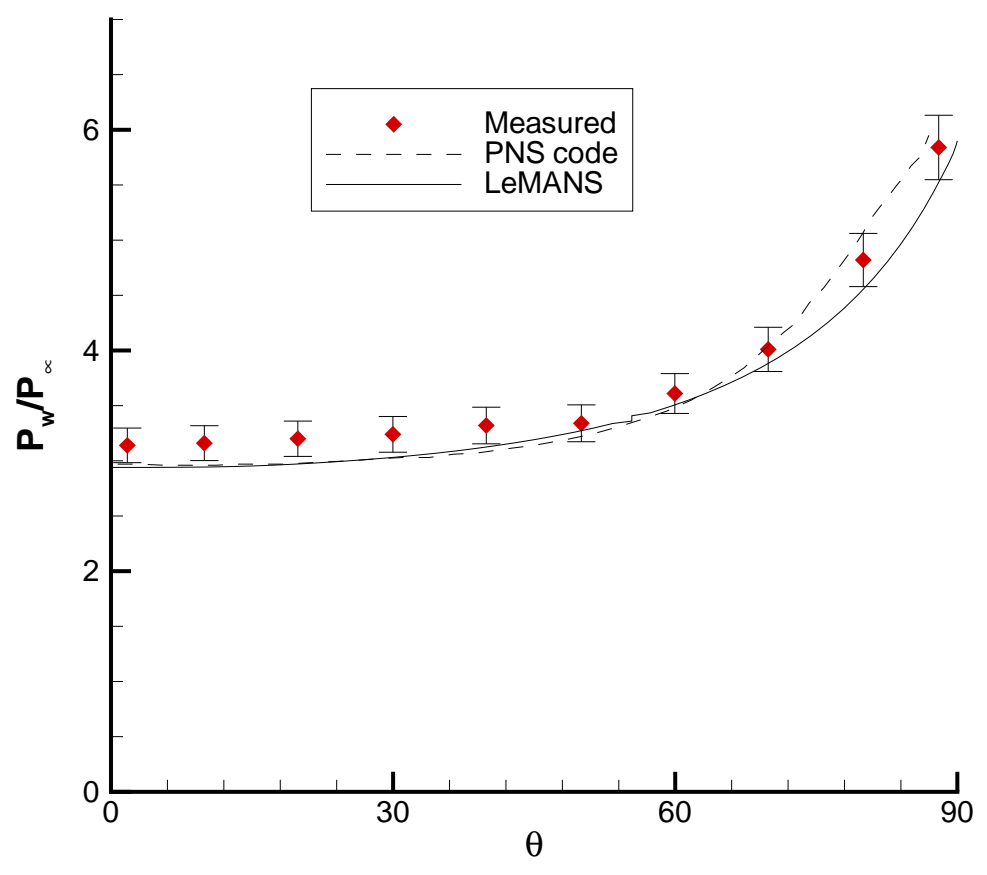

(a) Pressure around the circumference

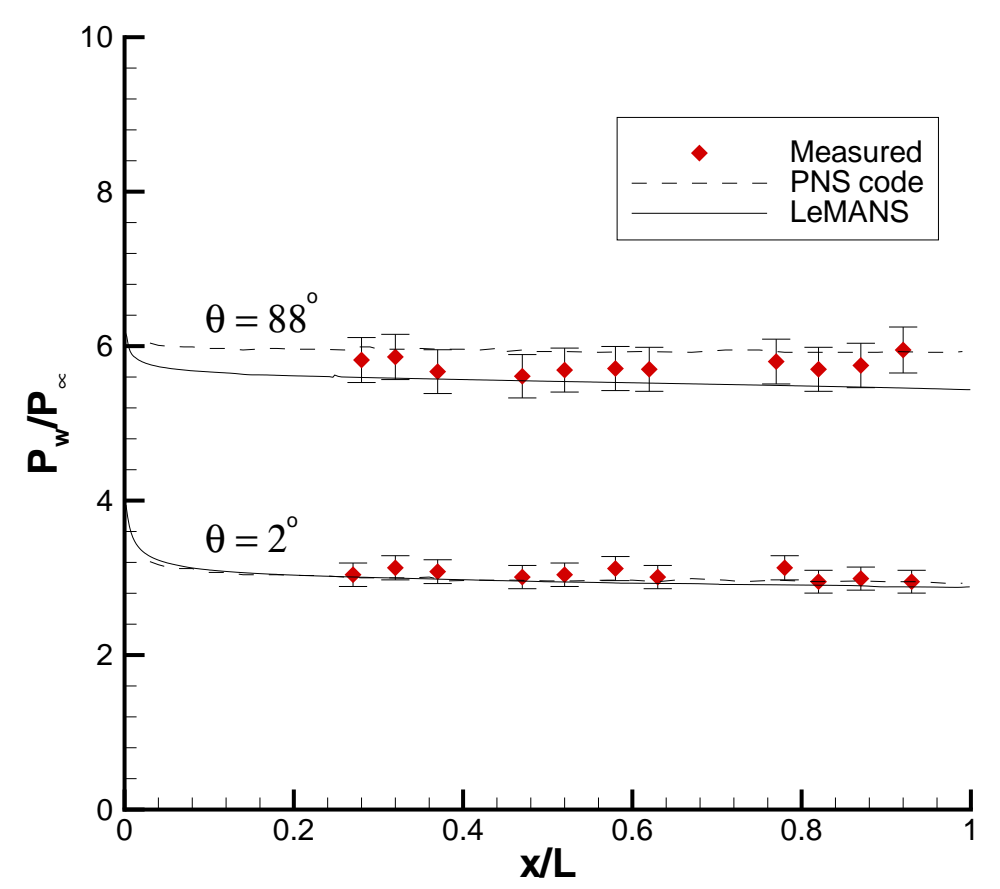

(b) Pressure along rays

Figure 3. Normalized surface pressure distributions for the Mach 8 sharp elliptic cone $\left( \pm 5\right.$ percent uncertainty) ${ }^{1}$ 


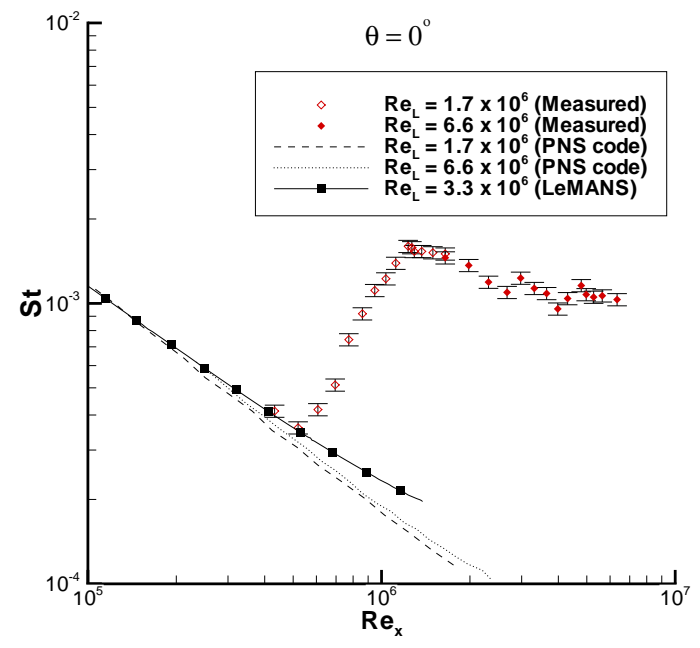

(a) Top centerline, $\theta=0^{\circ}$

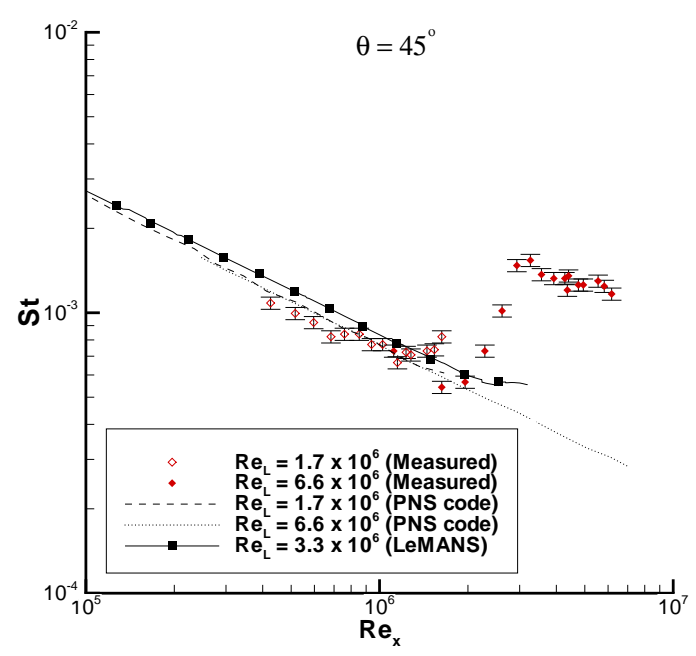

(b) Shoulder, $\theta=45^{\circ}$

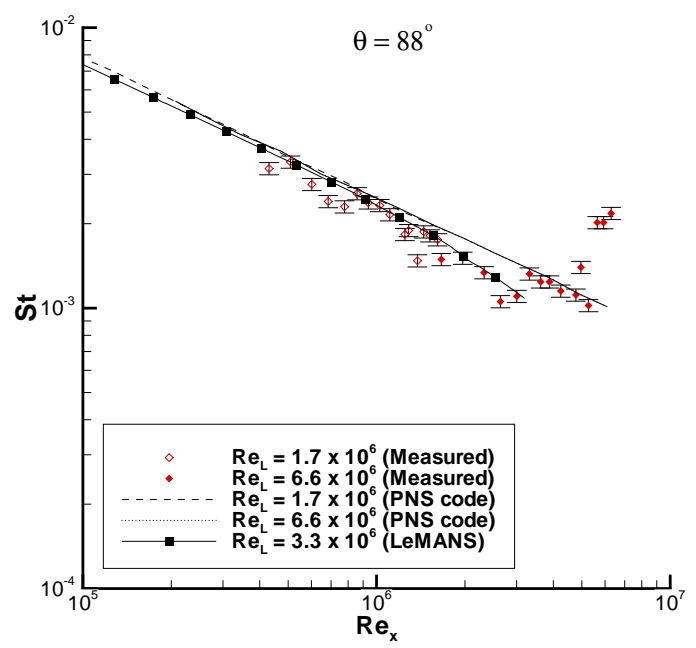

(c) Leading edge, $\theta=88^{\circ}$

Figure 4. Stanton number distributions for the Mach 8 sharp elliptic cone $( \pm 10$ percent uncertainty $){ }^{1}$

\section{2 of 23}



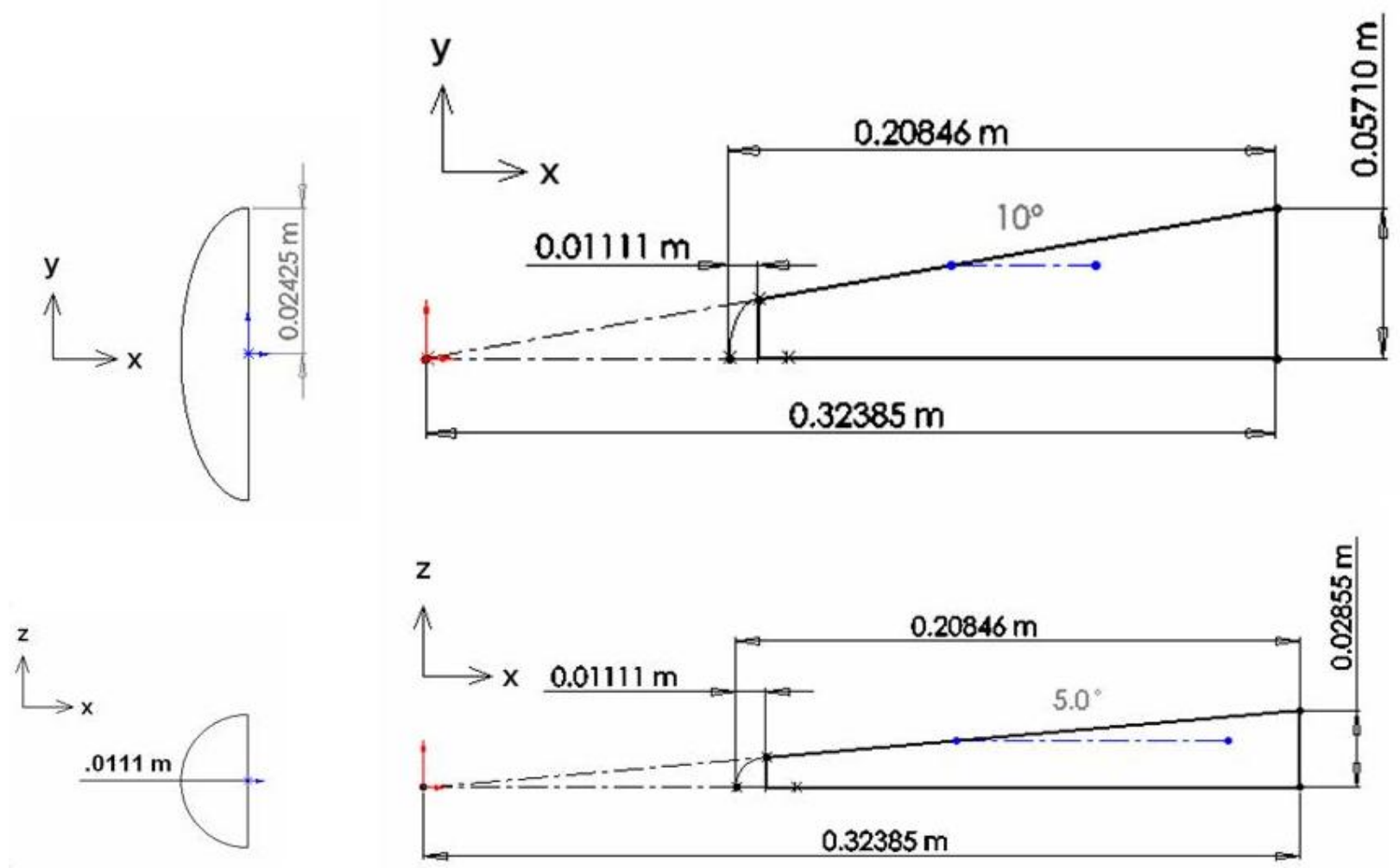

Figure 5. Blunt elliptic cone geometry. Dimensions are in meters. ${ }^{2}$ 


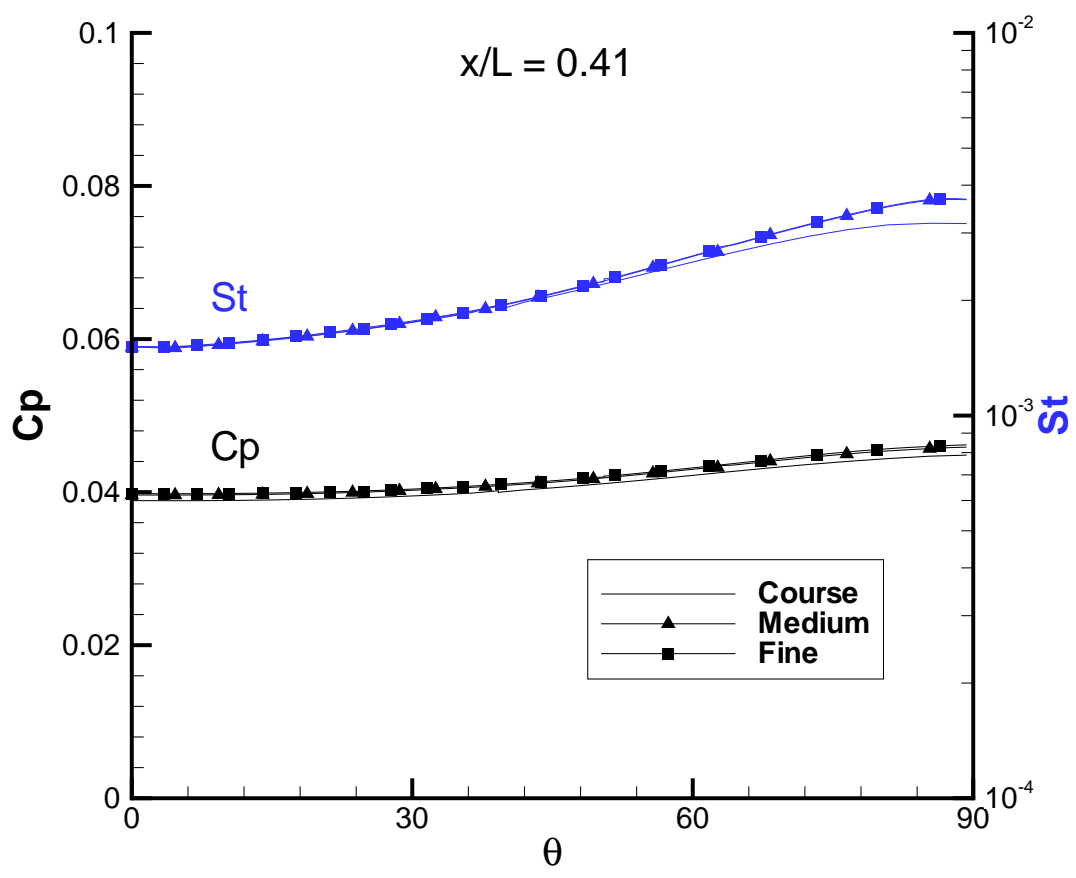

(a) Around the circumference

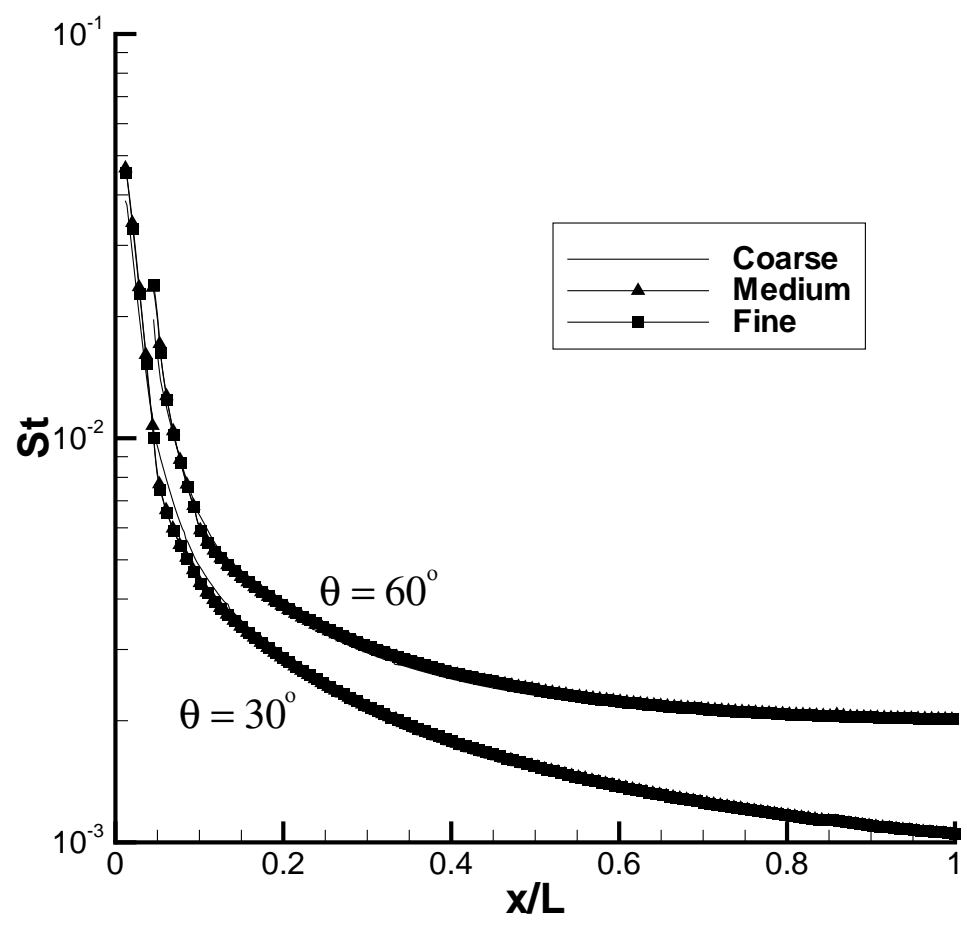

(b) Along rays

Figure 6. Grid independence study for Mach 14 blunt elliptic cone ( $L=0.2 \mathrm{~m})$. 


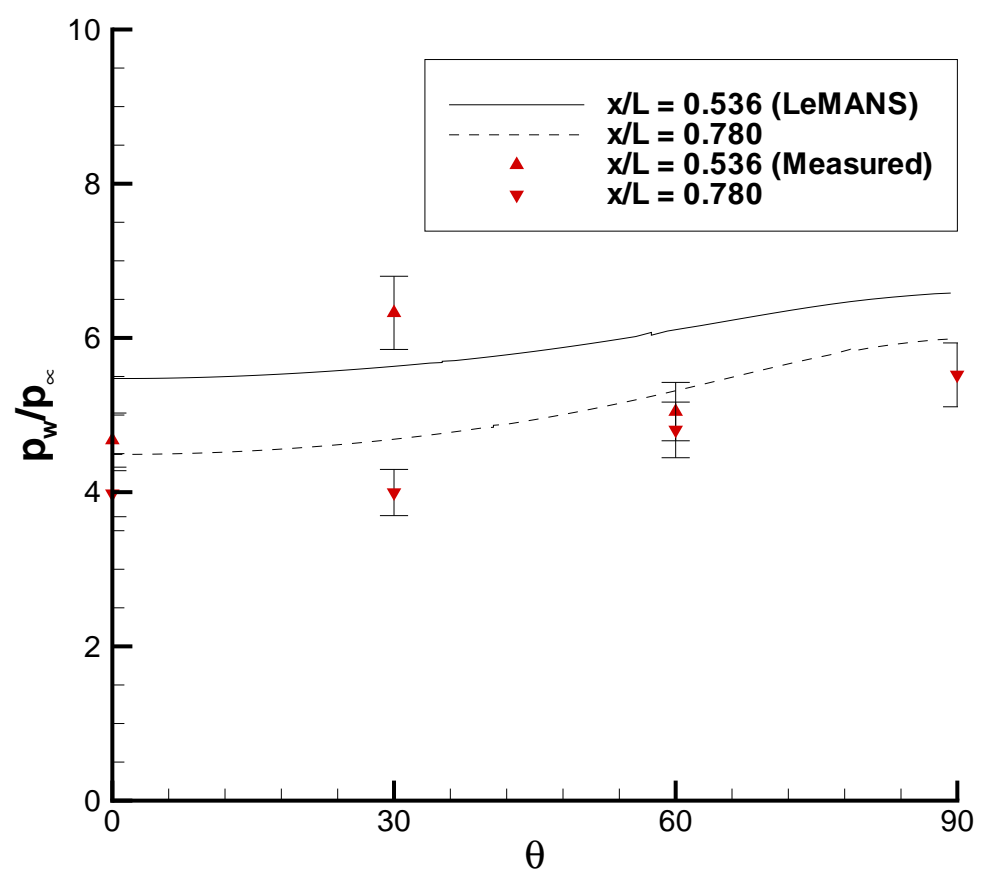

(a) Pressure around the circumference

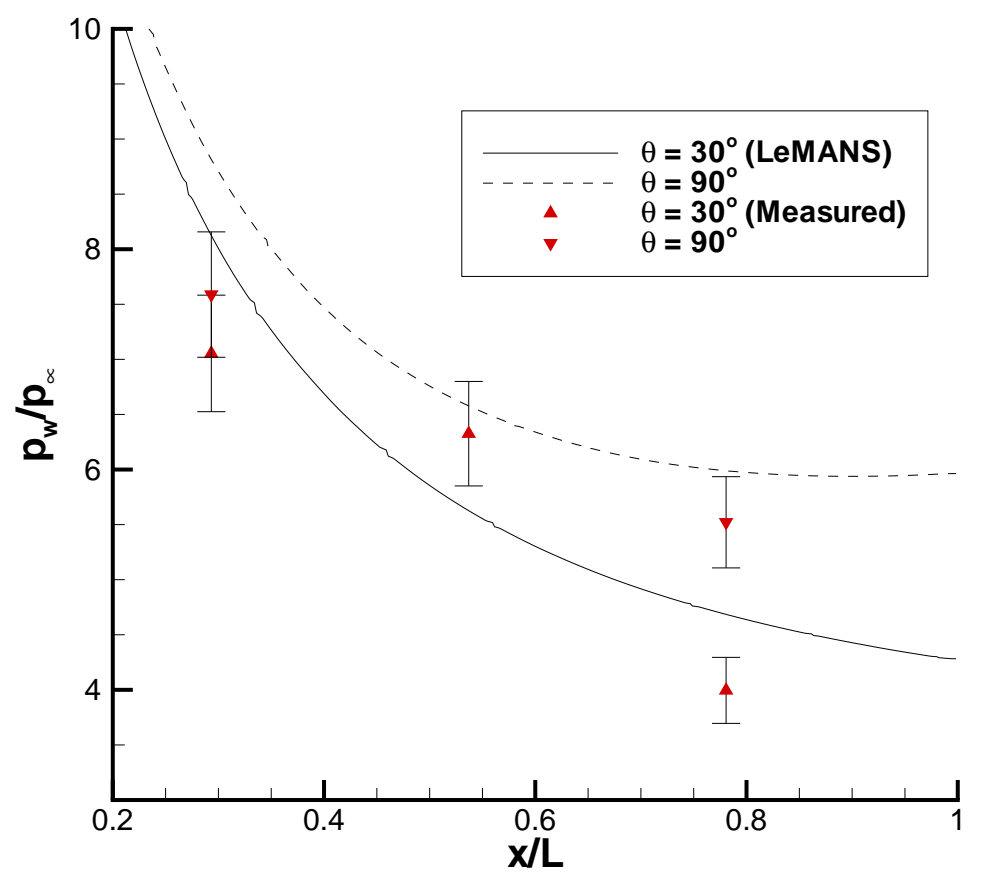

(b) Pressure along rays

Figure 7. Normalized surface pressure distributions for Mach 14 blunt elliptic cone $\left( \pm 7\right.$ percent uncertainty) ${ }^{2}$ 


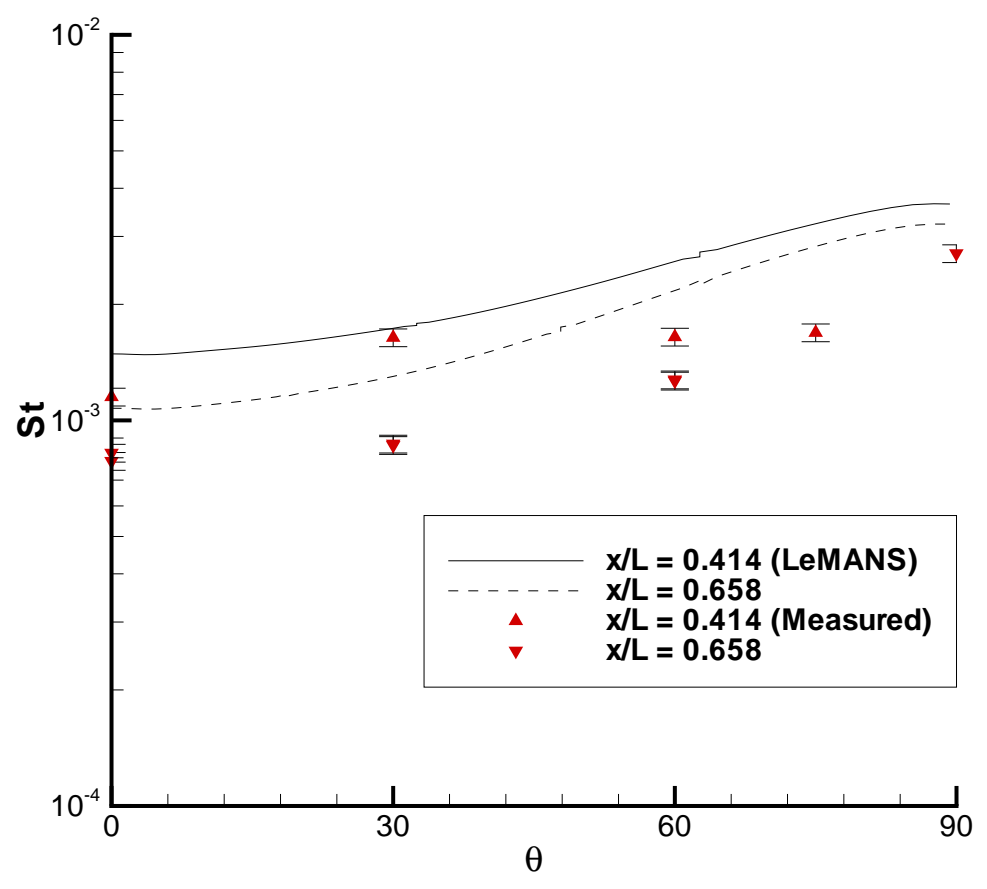

(a) Stanton number around the circumference

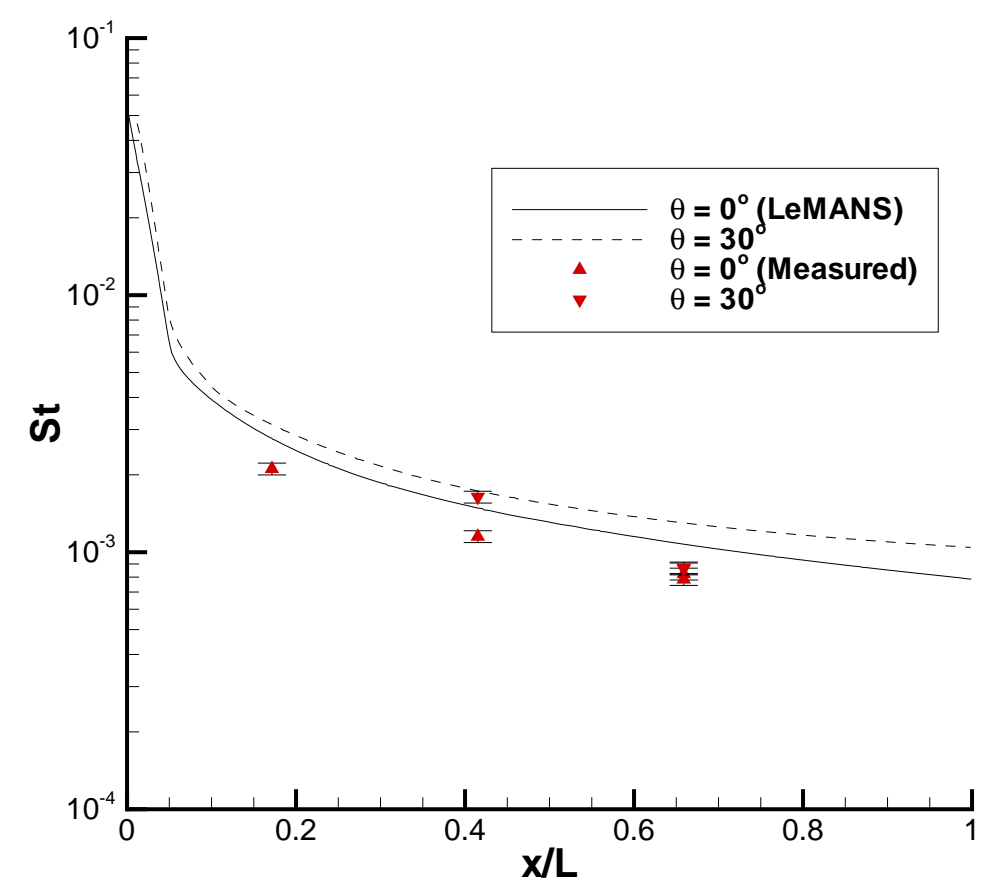

(b) Stanton number along rays

Figure 8. Stanton number distributions for Mach 14 blunt elliptic cone $( \pm 4.5$ percent uncertainty $){ }^{2}$ 


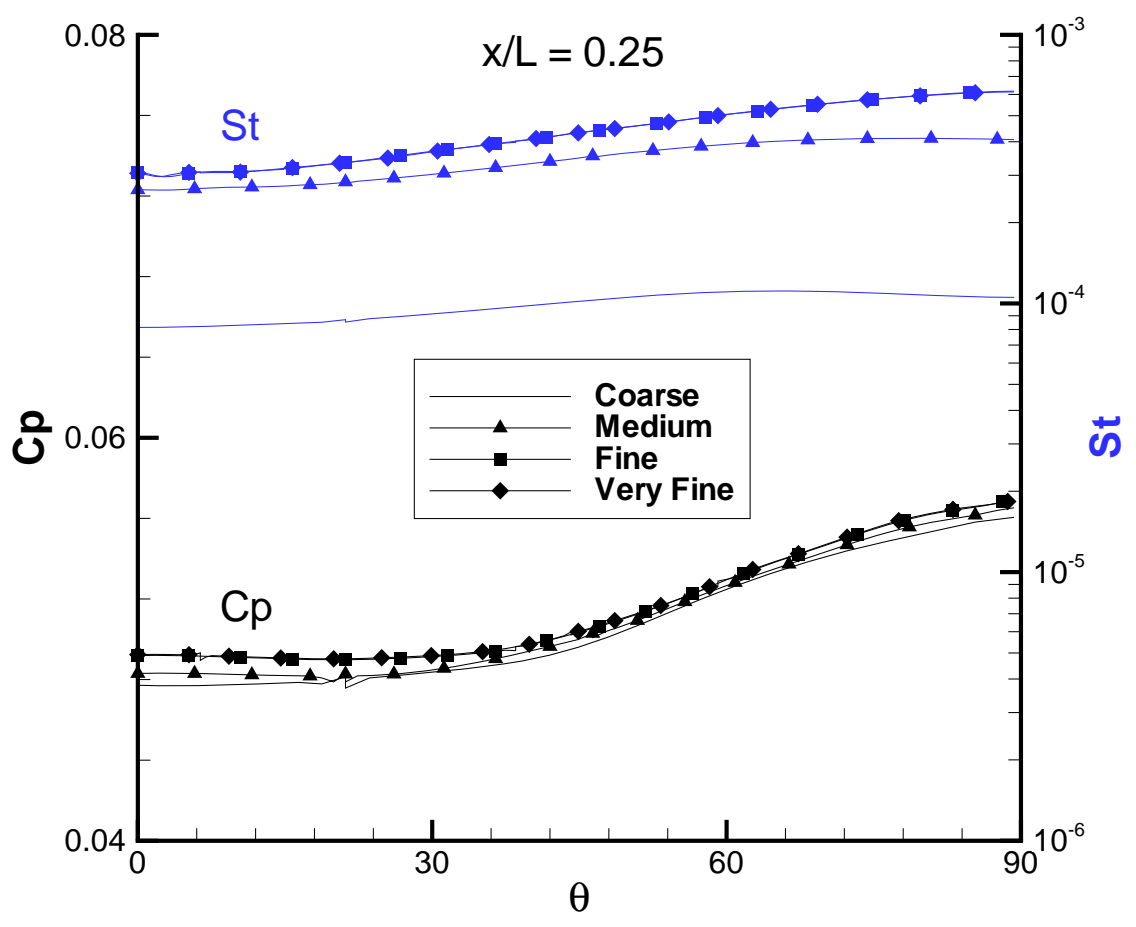

(a) Around the circumference

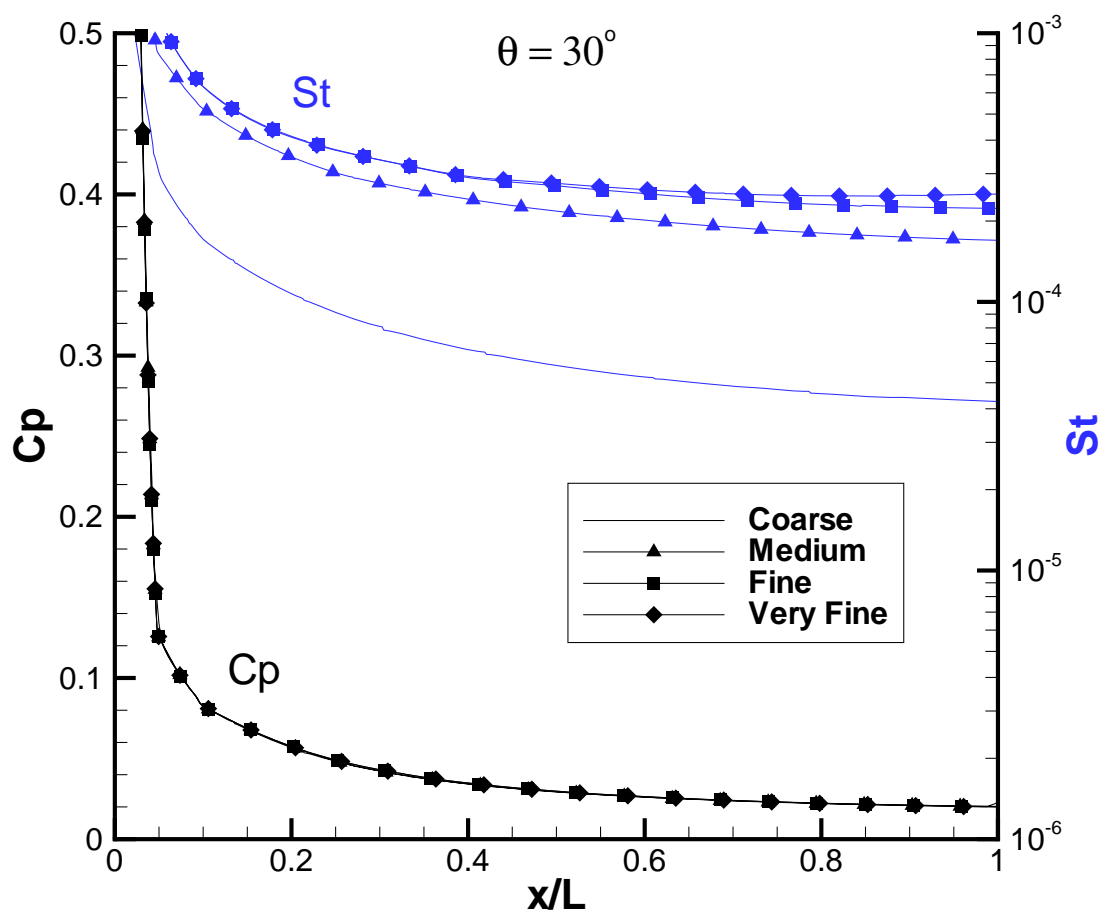

(b) Along rays

Figure 9. Grid independence study for Mach 12 blunt elliptic cone ( $L=3 \mathbf{m})$. 


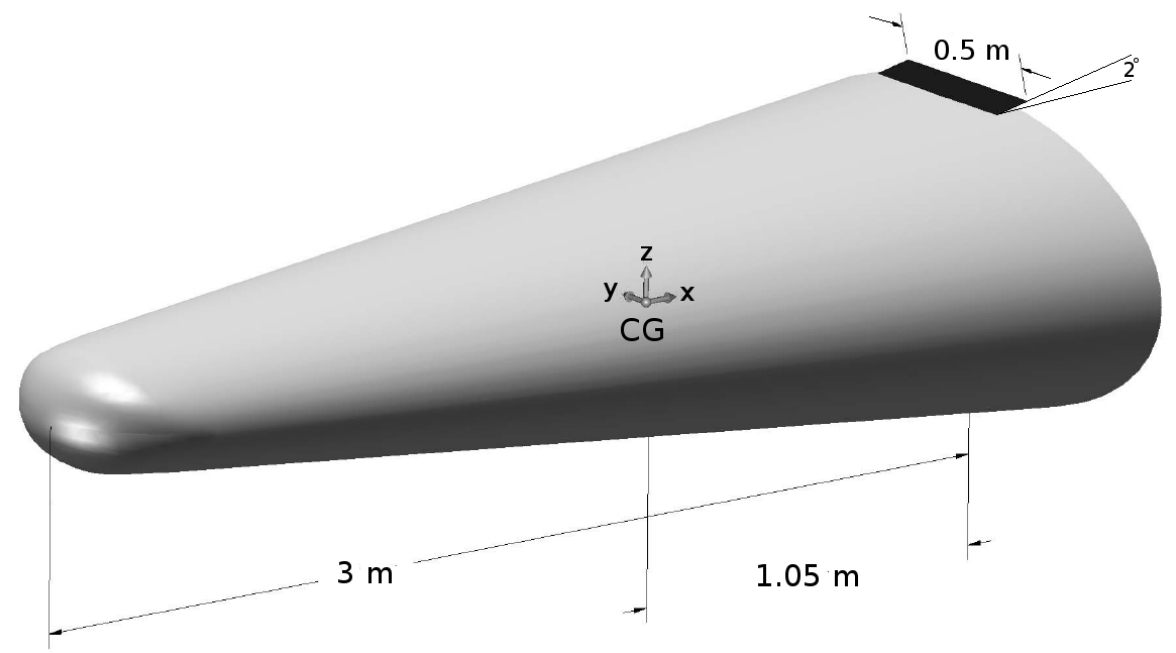

Figure 10. Model of a $2^{\circ}$ mechanical flap attached to a blunt elliptic body.

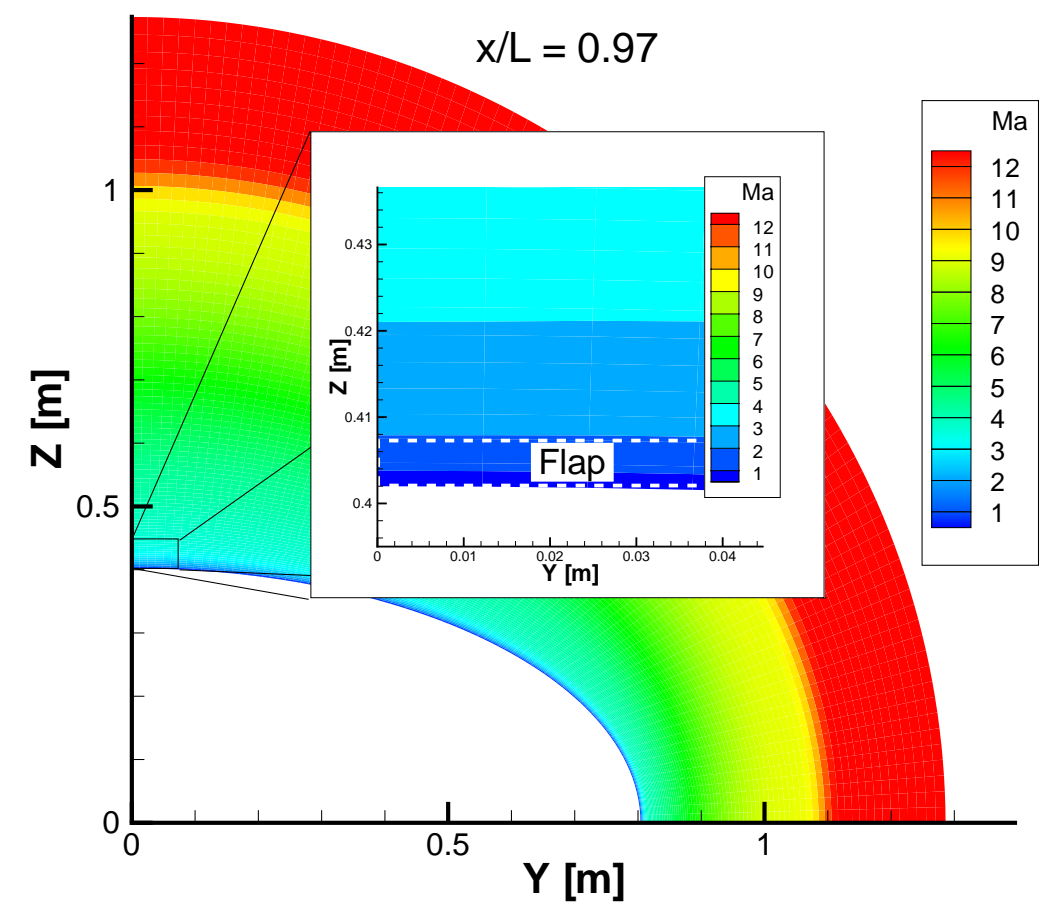

Figure 11. Mach profile at $\mathrm{x} / \mathrm{L}=0.97$ for Mach 12 blunt elliptic cone $(L=3 \mathbf{m})$. 


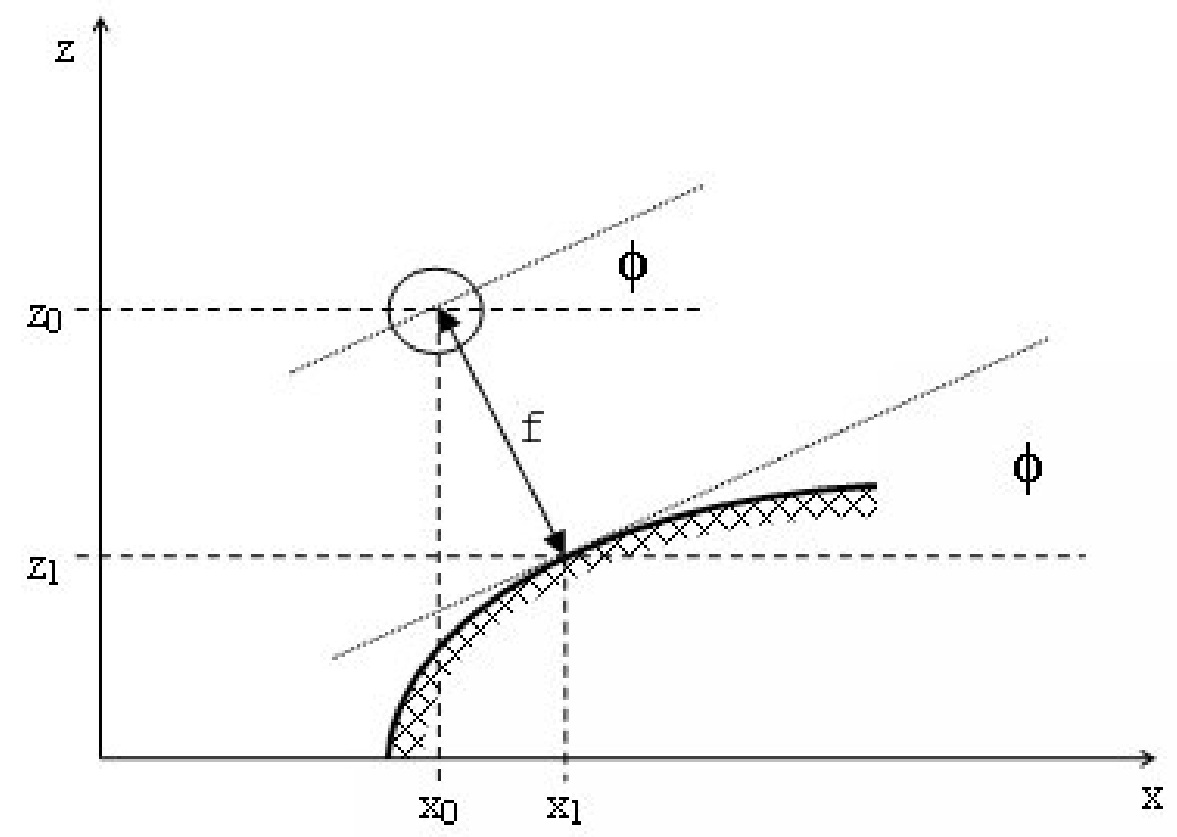

Figure 12. Illustration of the constant height parameter used to determine $z_{0}$ and $\phi$ for parametric study.

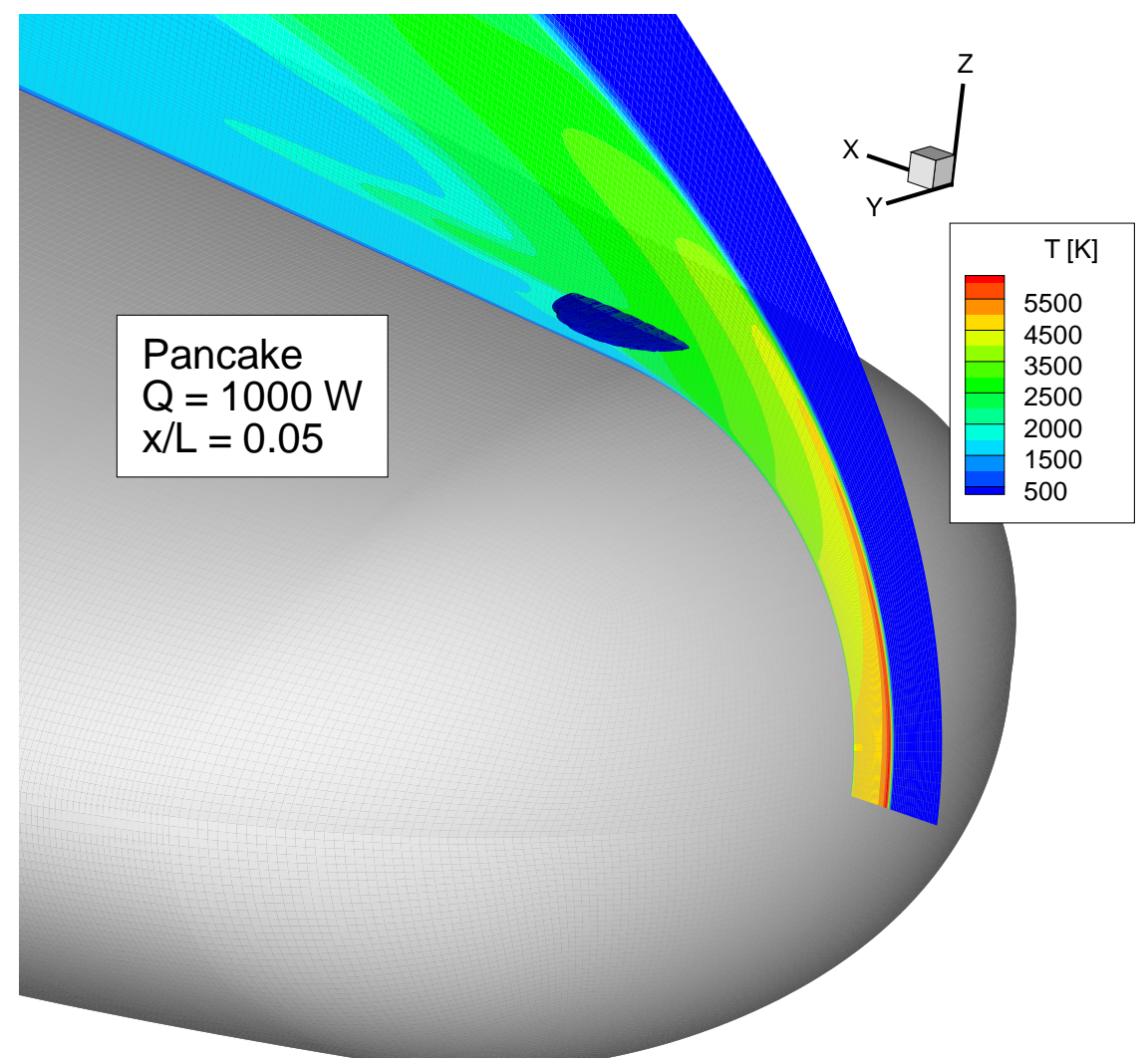

Figure 13. Slice of the temperature contour along the top centerline for a pancake deposition. 


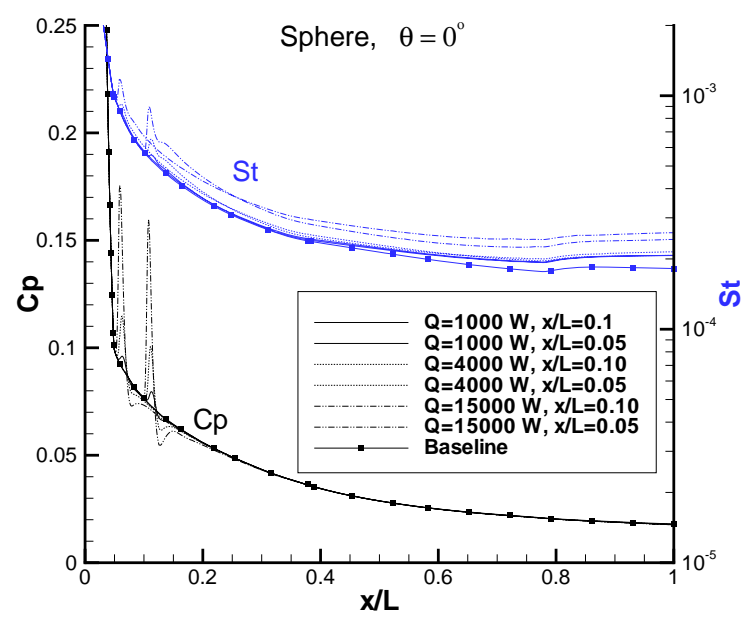

(a) Sphere deposition

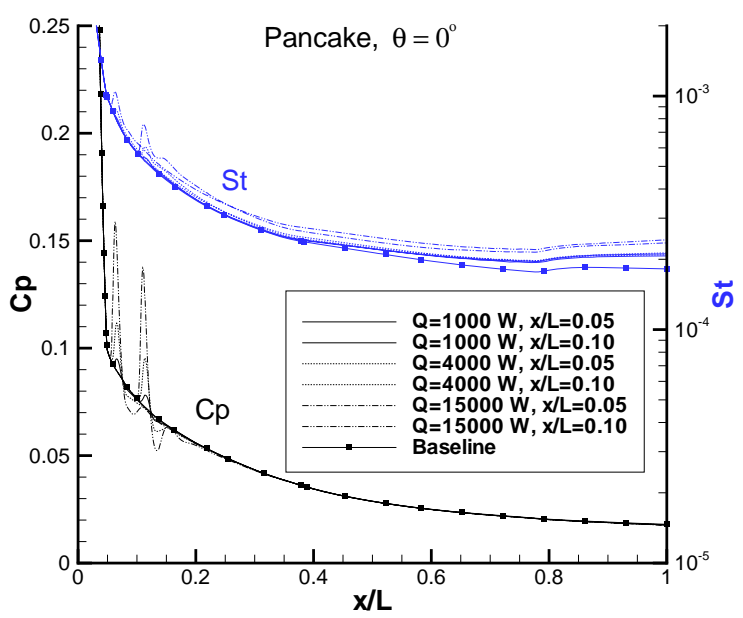

(b) Pancake deposition

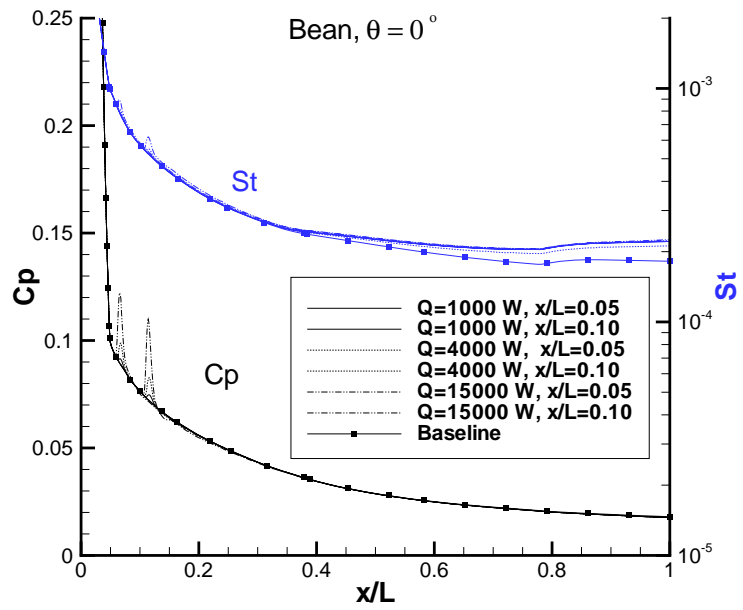

(c) Bean deposition

Figure 14. Pressure coefficient and Stanton number distributions for the Mach 12 blunt elliptic cone $(L=3 \mathrm{~m})$ for various energy deposition patterns.

\section{0 of 23}




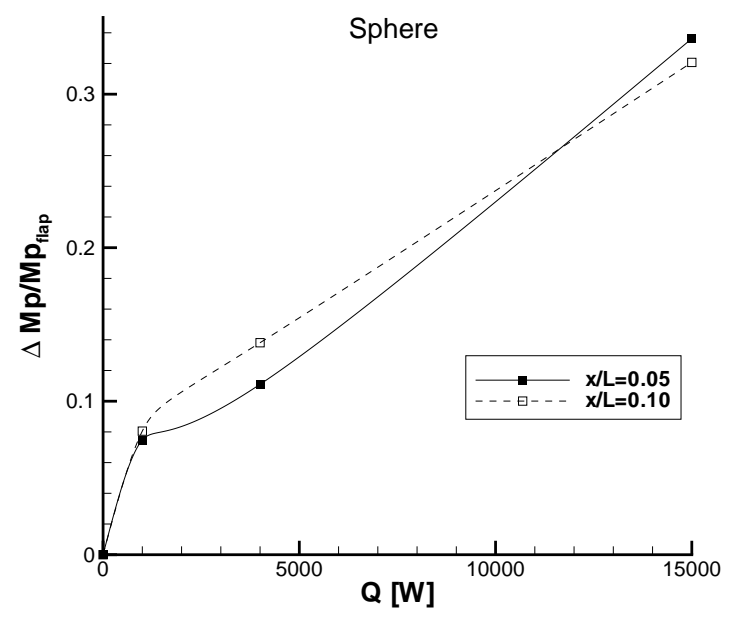

(a) Sphere deposition

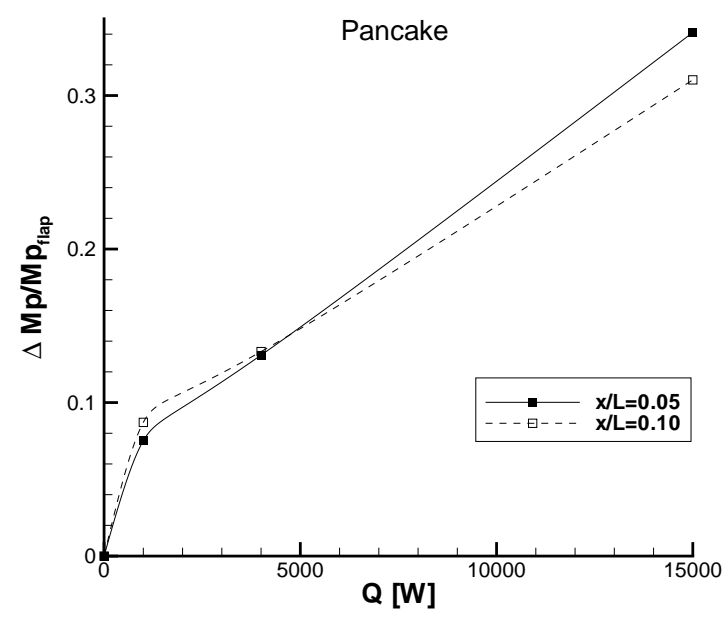

(b) Pancake deposition

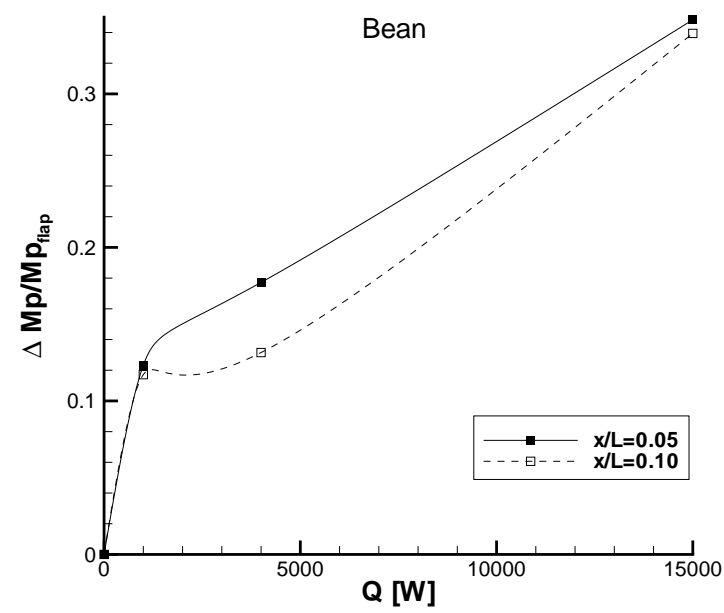

(c) Bean deposition

Figure 15. Normalized change in pitching moment for a Mach 12 blunt elliptic cone $(L=3 \mathrm{~m})$ for various energy deposition patterns. 


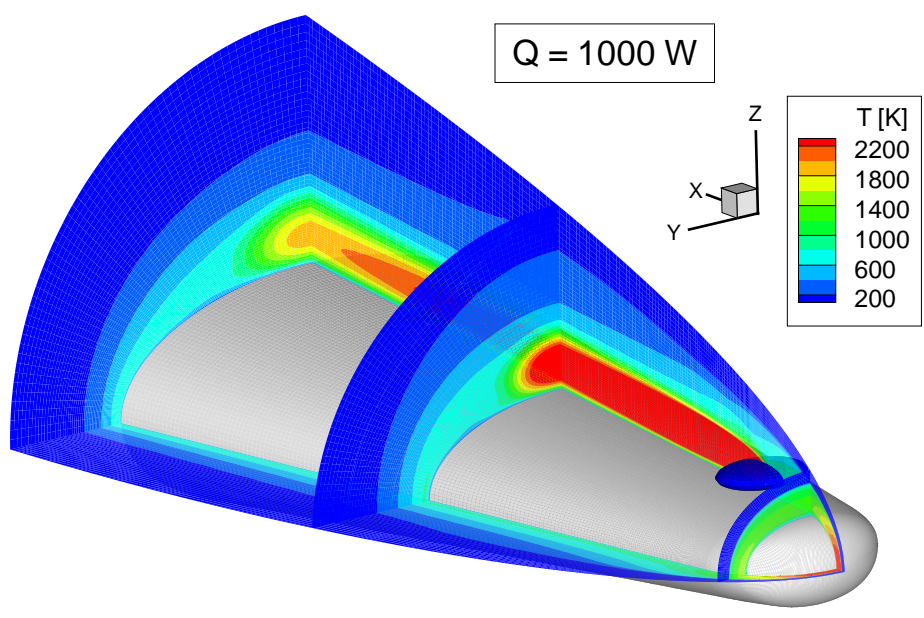

Figure 16. Temperature contours for blunt elliptic cone $(L=0.2 \mathbf{m})$ with $Q=1000 \mathrm{~W}$.

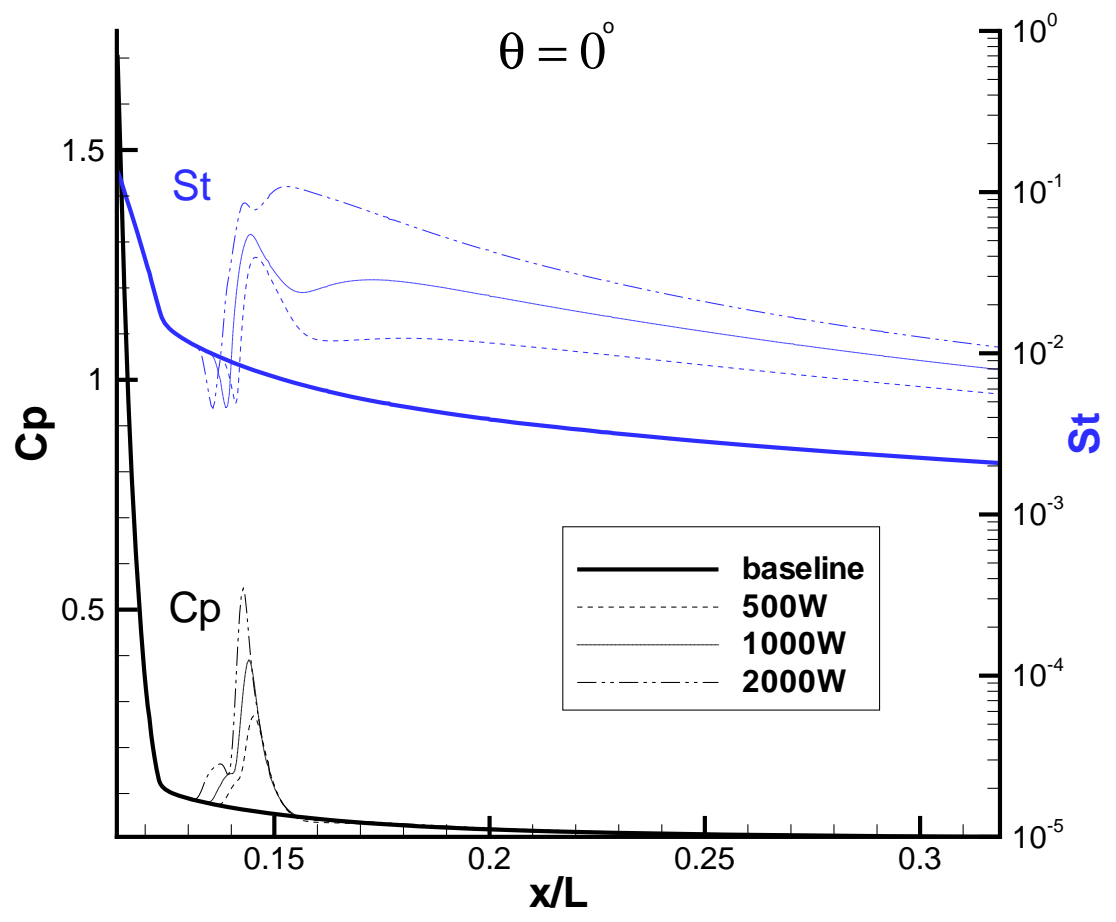

Figure 17. Pressure coefficient and Stanton number distributions along the top center line of a Mach 14 blunt elliptic cone $(L=0.2 \mathrm{~m})$ with various amounts of energy deposition 


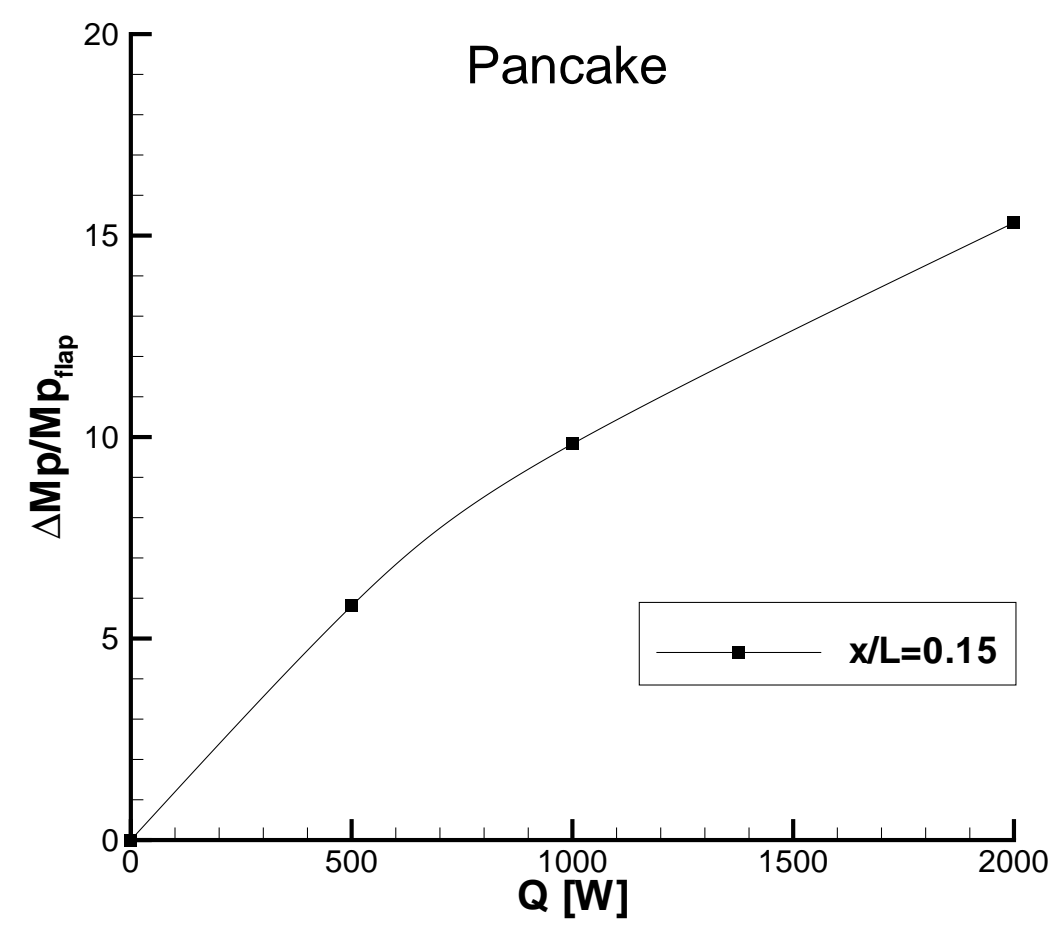

Figure 18. Normalized change in pitching moment for a Mach 14 blunt elliptic cone $(L=0.2$ m) for a pancake energy deposition.

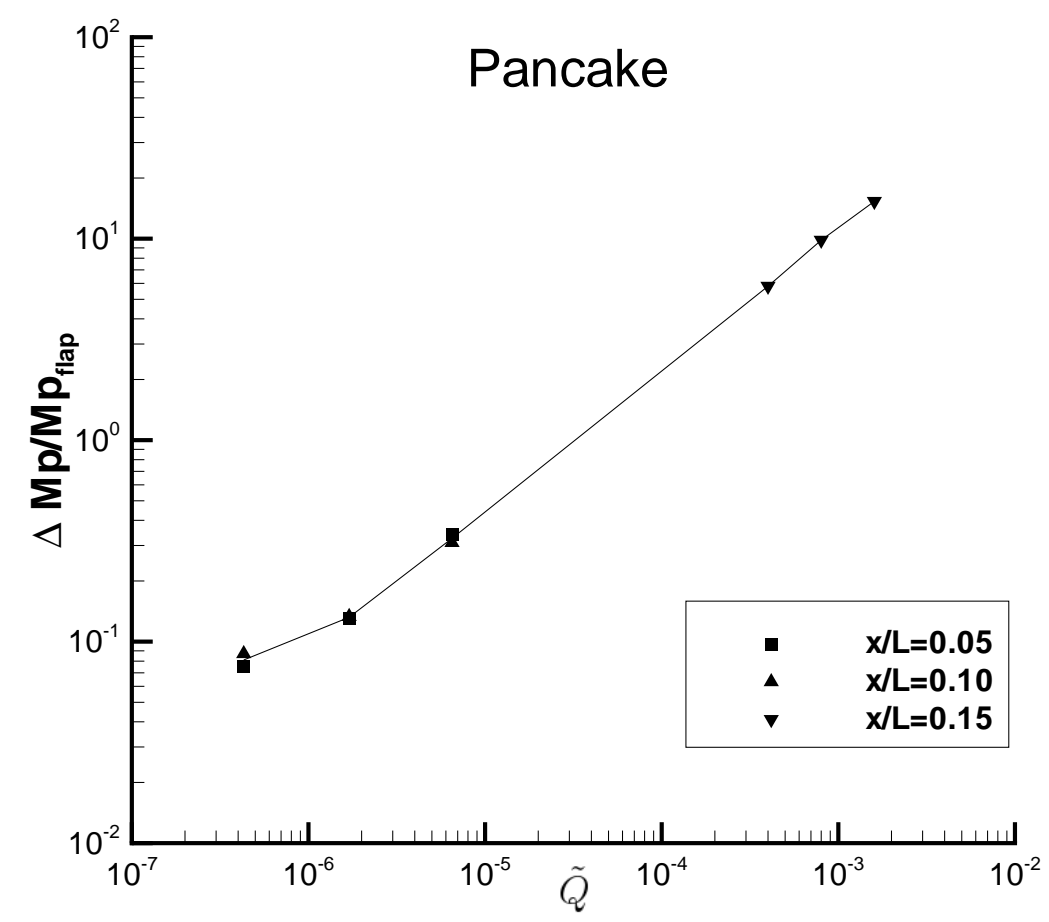

Figure 19. Normalized change in pitching moment versus the non-dimensional total energy deposition $\tilde{Q}$ for a pancake energy deposition.

23 of 23 